\title{
Translation of Chemical Structure into Dissipative Particle Dynamics Parameters For Simulation of Surfactant Self- Assembly.
}

Ennio Lavagnini, ${ }^{\mathrm{a}}$ Joanne L. Cook, ${ }^{\mathrm{b}}$ Patrick B. Warren, ${ }^{\mathrm{b}, \mathrm{c}}$ and Christopher A. Hunter ${ }^{\text {a* }}$

${ }^{a}$ Department of Chemistry, University of Cambridge, Lensfield Road, Cambridge CB2 1EW, United Kingdom.

${ }^{\mathrm{b}}$ Unilever R\&D Port Sunlight, Quarry Road East, Bebington CH63 3JW, United Kingdom.

c The Hartree Centre, STFC Daresbury Laboratory, Warrington WA4 4AD, United Kingdom.

\section{Supplementary Information}

Density Functional Theory calculations

Determination of $\boldsymbol{N}_{\text {cut }}$

2D histograms of assembly shape distribution

S4

Parameters for bonded interactions

S13

Amide conformers

S26 


\section{Density Functional Theory Calculations}

DFT calculations were carried out with Gaussian9-D01 with B3LYP/631G*. The molecules were constructed using molecular mechanics, the structures were optimised using DFT, and the MEPS was calculated on the 0.002 electron Bohr ${ }^{-3}$ electron density isosurface.

Footprinting of the DFT MEPS was carried out using the method described in Calero, C. S.; Farwer, J.; Gardiner, E. J.; Hunter, C. A.; Mackey, M.; Scuderi, S.; Thompson, S.; Vinter, J.G. Footprinting Molecular Electrostatic Potential Surfaces for Calculation of Solvation Energies. Phys. Chem. Chem. Phys, 201315 (41), 18262.

https://doi.org/10.1039/c3cp53158a (internal git version 44285baa). The resulting SSIP distribution for each molecule is shown in Figure S3 and the values of the SSIPs are given in Table 1 of the main text.

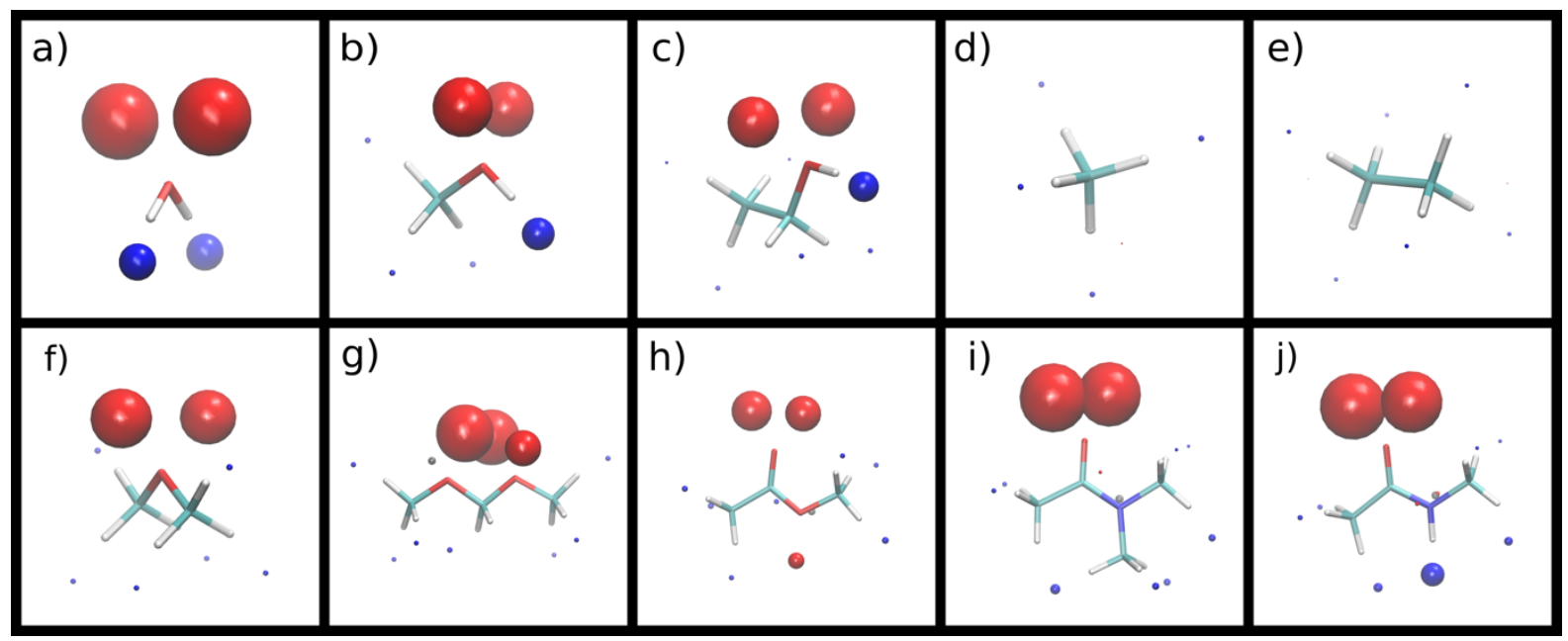

Figure S1: SSIPs calculated by footprinting the electrostatic potential calculated on 0.002 Bohr $\AA^{-3}$ electron density isosurfaces calculated using DFT and B3LYP/631G*. (a) water (b) methanol (c) ethanol (d) methane (e) ethane (f) methoxymethane (g) dimethoxymethane (h) methyl acetate (i) N,N-dimethyl acetamide (j) N-methyl acetamide.

Solvation energies were calculated using the SSIMPLE method described in Hunter, C. A. A Surface Site Interaction Model for the Properties of Liquids at Equilibrium. Chem. Sci. 2013, 4 (4), 1687. https://doi.org/10.1039/c3sc22124e (internal git version 9ae19fda).

DPD simulations were run on a GNU/Linux centos 6.10 system powered by two eightcore Intel Xeon E5-2650 v2 (2.60GHz, Ivy Bridge) CPUs with 128GB of RAM. 


\section{Determination of $\boldsymbol{N}_{c u t}$}

(a)

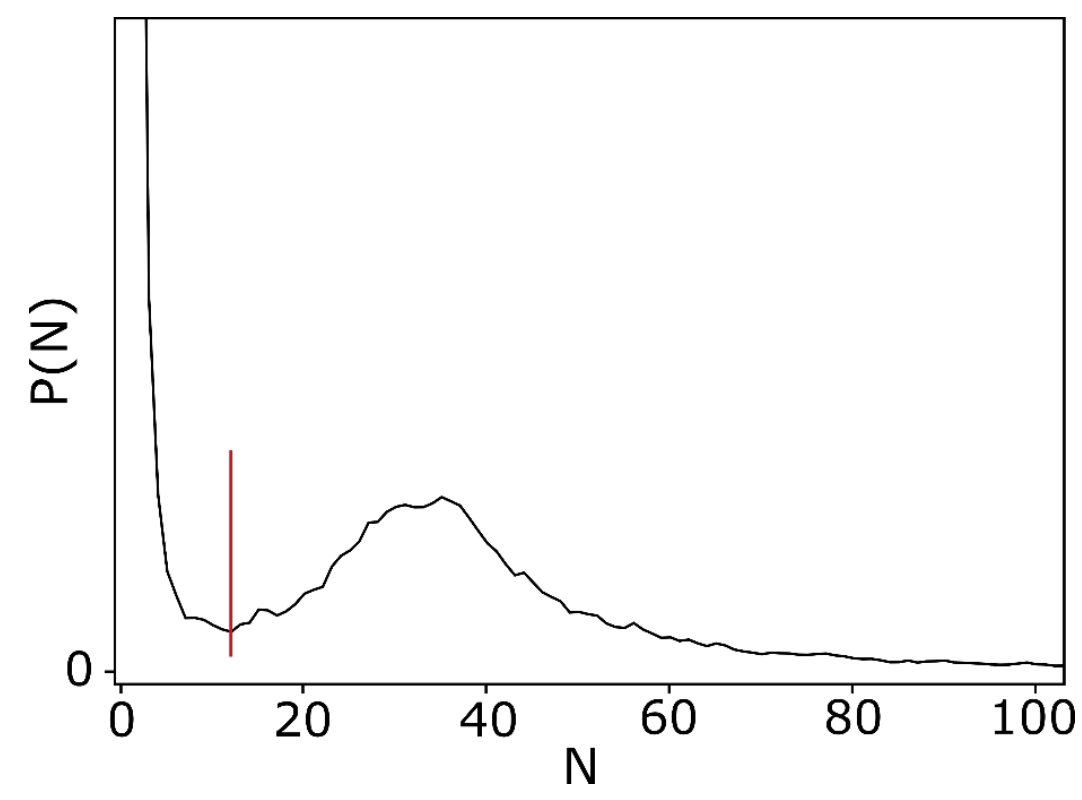

(b)

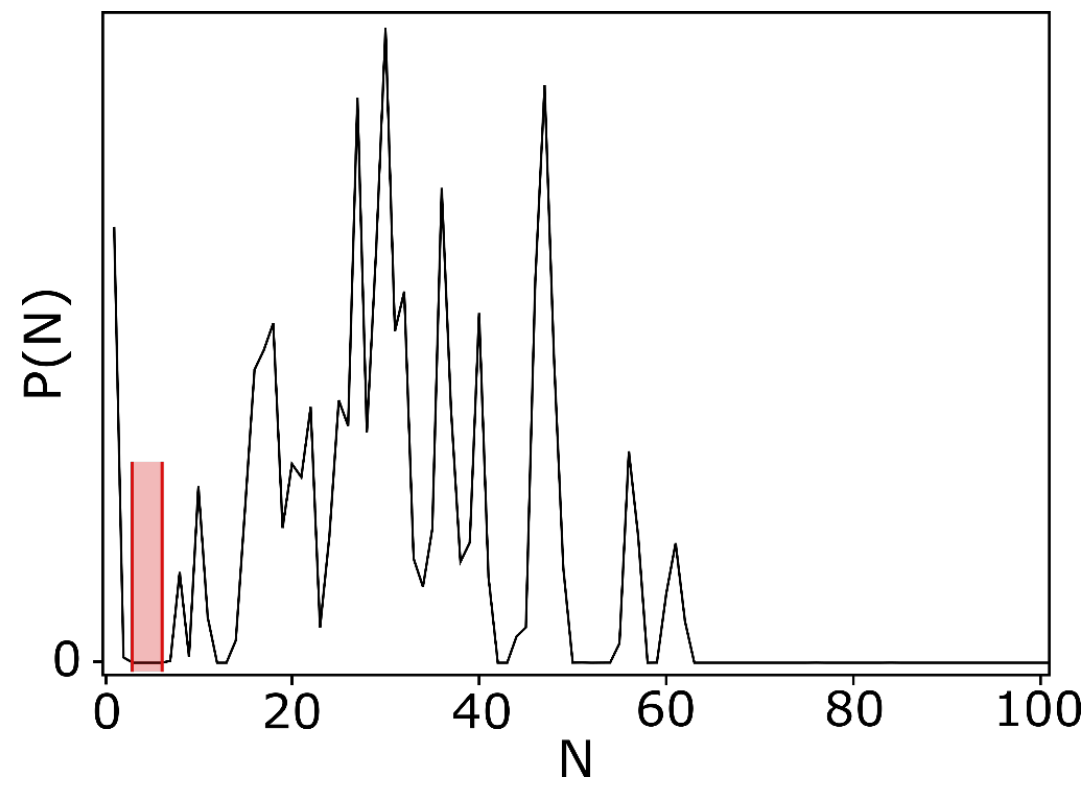

Figure S1: (a) Aggregation distribution (N) for MEA8 surfactant at 5\%wt. Ncut is highlighted in red. (b) Aggregation distribution (N) for MALTO12 surfactant at 5\%wt. Ncut gap is highlighted in red. As we have shown previously, this kind of jagged $P(N)$ distribution does not significantly affect the accuracy of the values of CMC and $N_{\text {agg }}$ that are calculated from it (publication (see J. Phys. Chem. B 2020, 124, 5047)). 


\section{D histograms of assembly shape distribution}

\section{GLY histograms}

a)

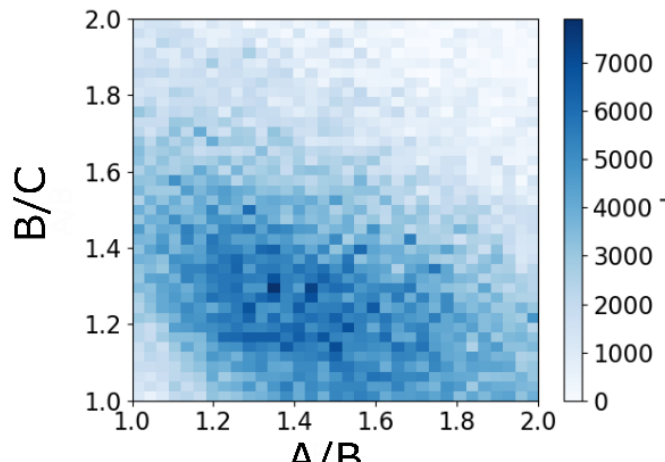

c) A/B

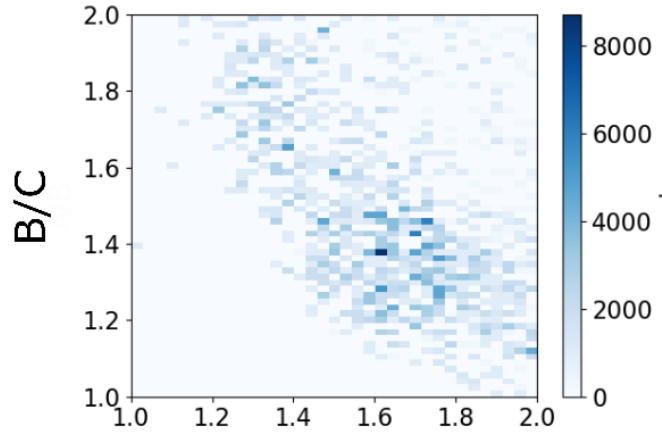
b)

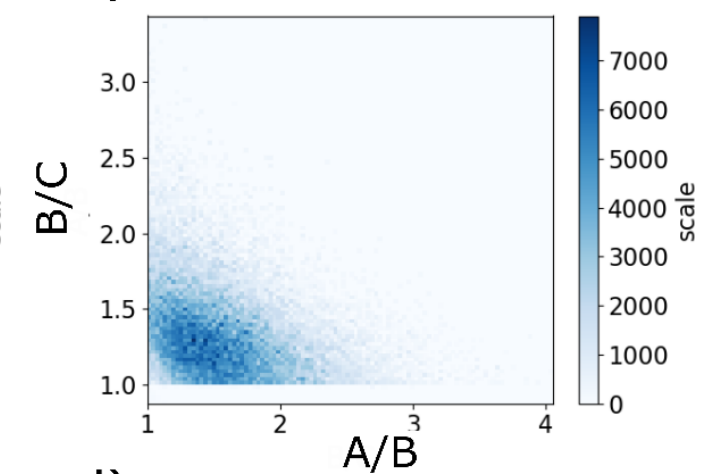

e)

A/B

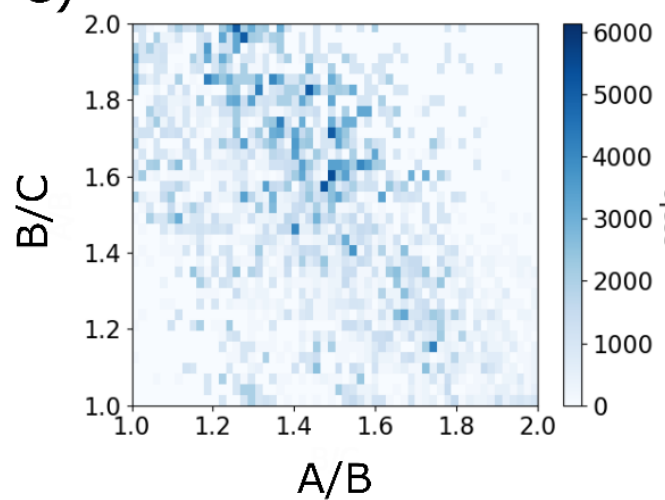

d)

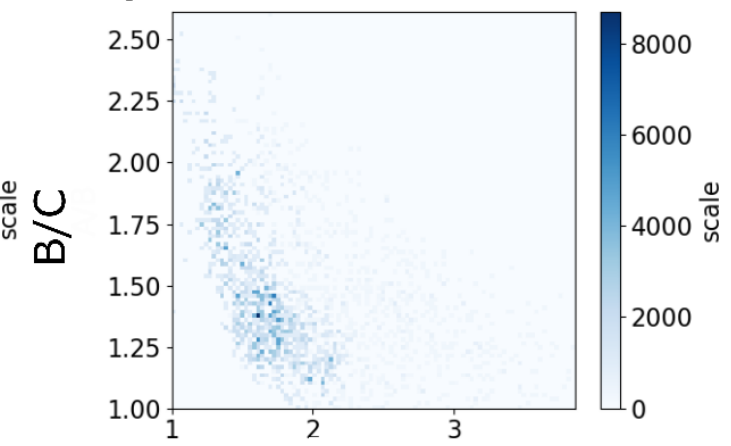

f)

A/B

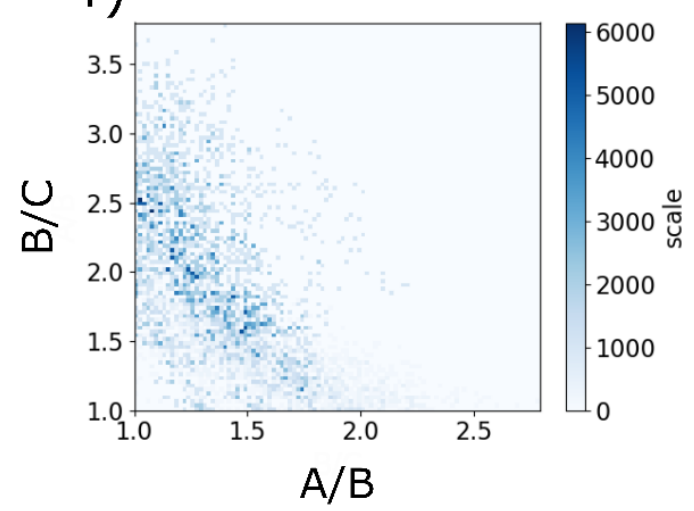

Figure S2: Histograms for GLY systems. a-b) GLY8 histograms. c-d) GLY10 histograms. e-f) GLY12 histograms. 


\section{XYL histograms}

a)

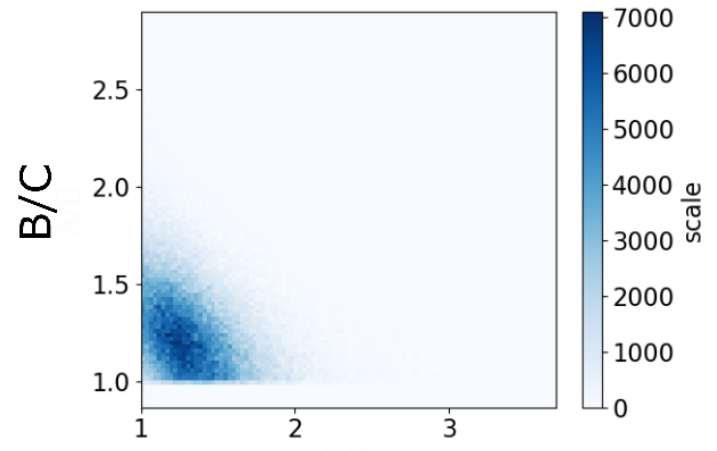

\section{c)}
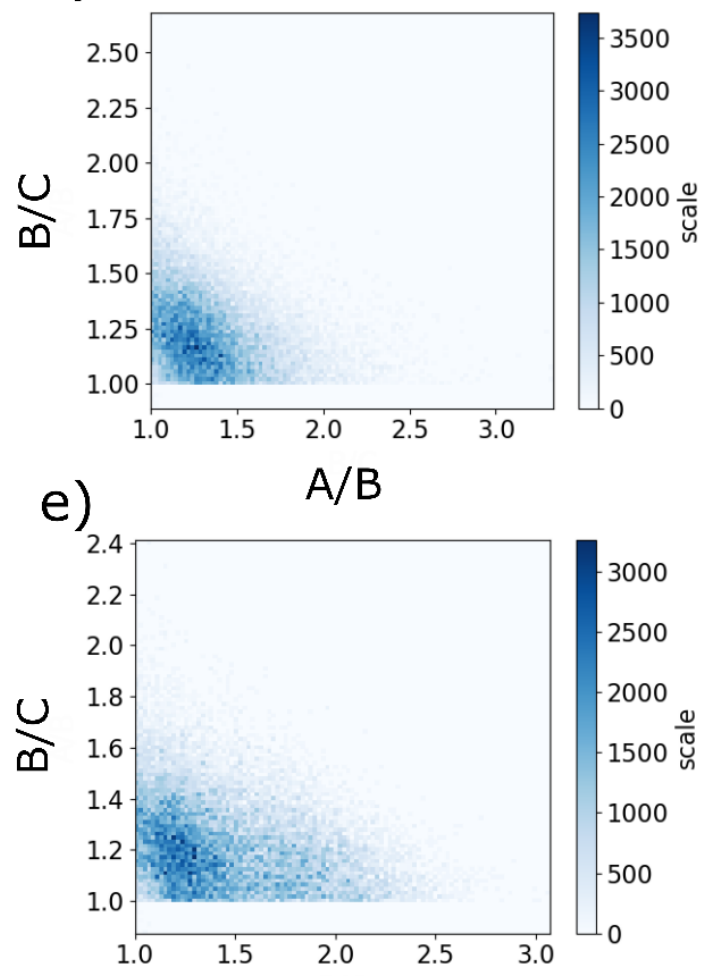

A/B b)

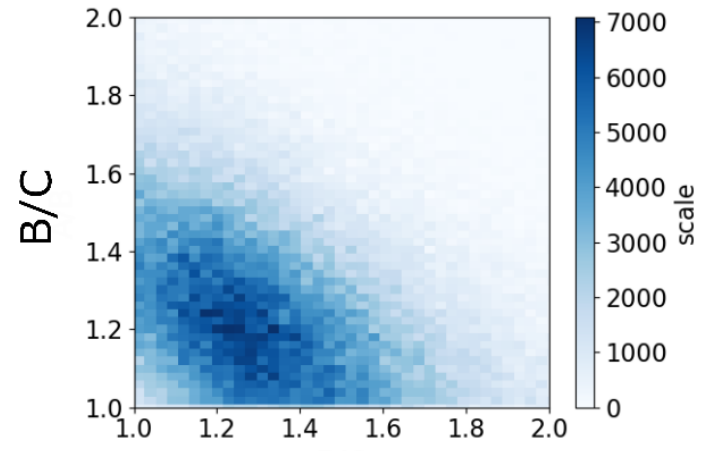

d)
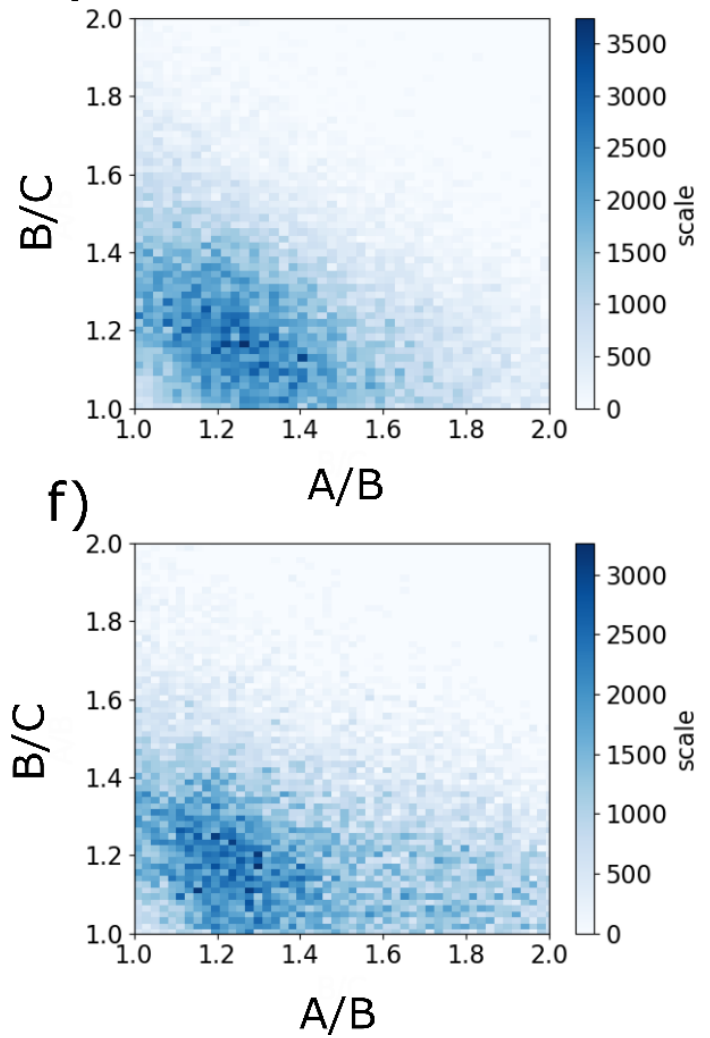

Figure S3: Histograms for XYL systems. a-b) XYL8 histograms. c-d) GLY10 histograms. e-f) GLY12 histograms. 


\section{MEA histograms}

a)

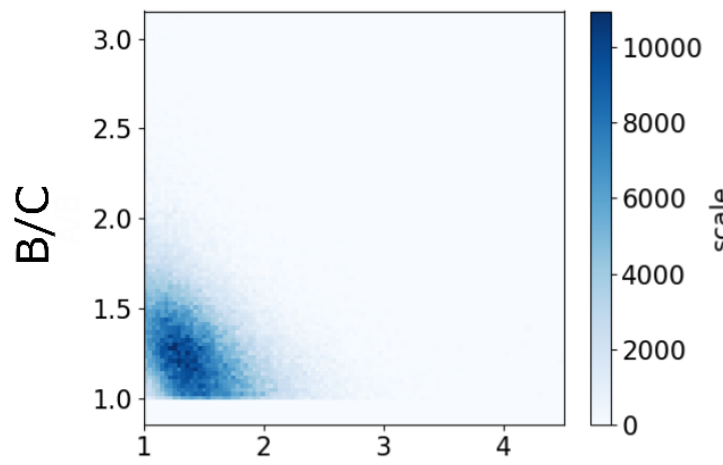

c)

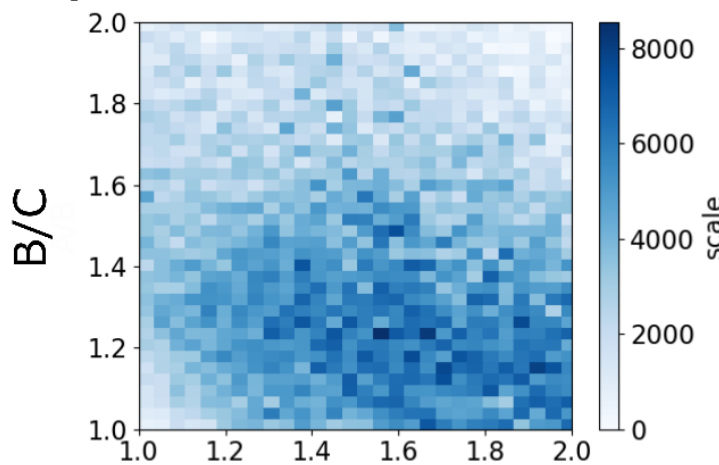

e)

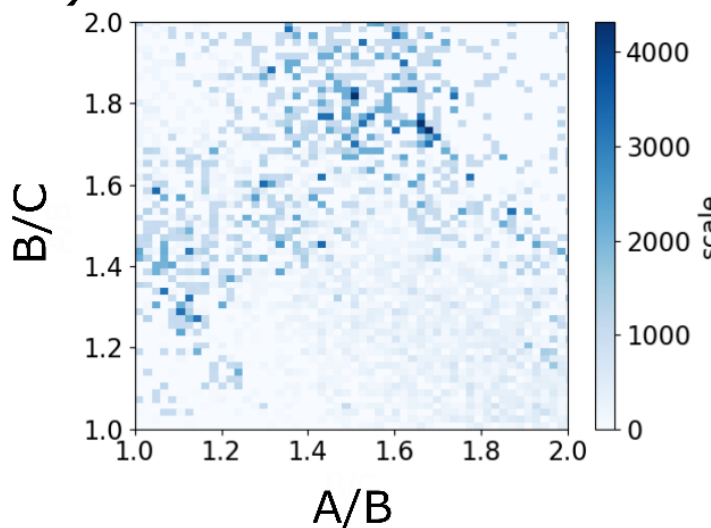

b)

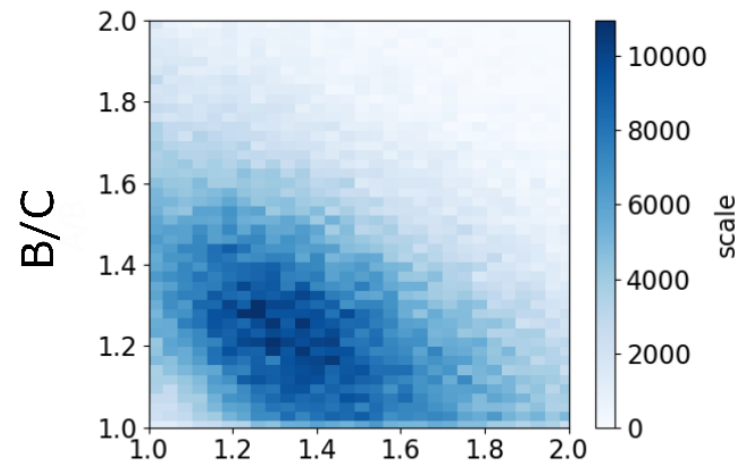

d)
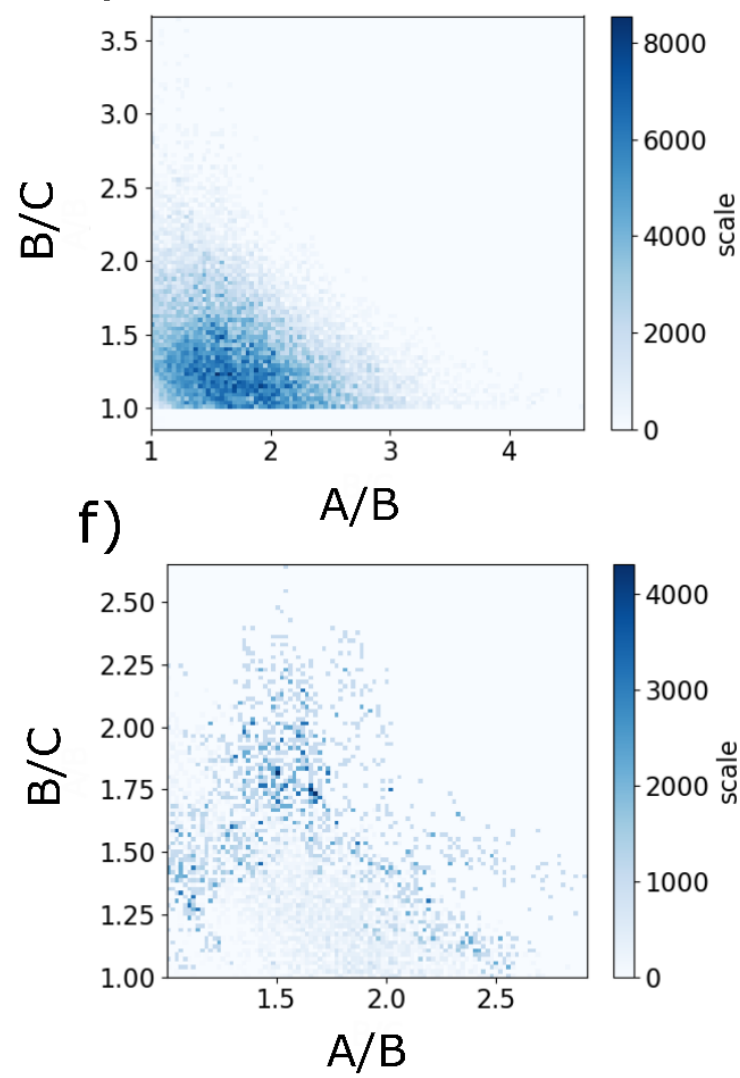

Figure S4: Histograms for MEA systems. a-b) MEA8 histograms. c-d) MEA10 histograms. e-f) MEA12 histograms. 
a)

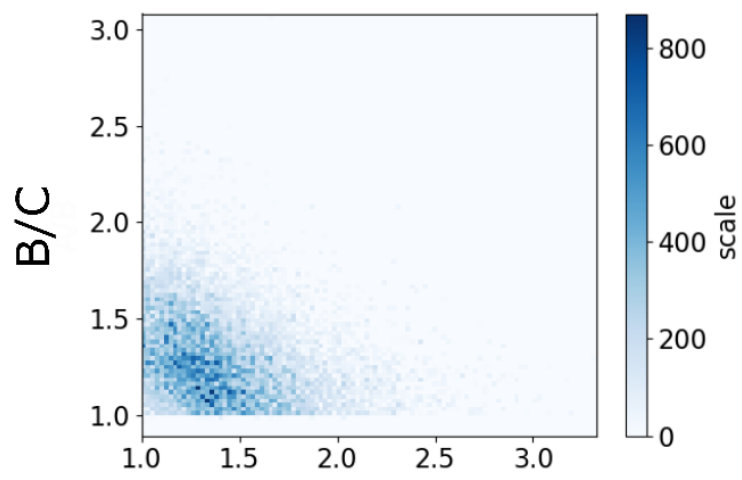

c)

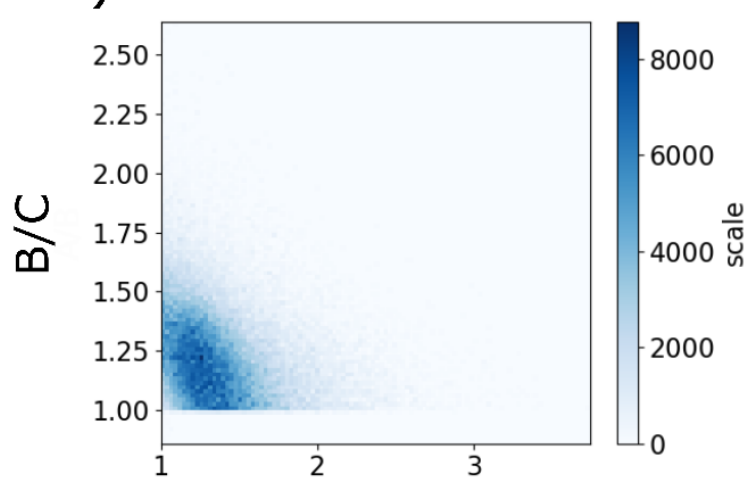

e)

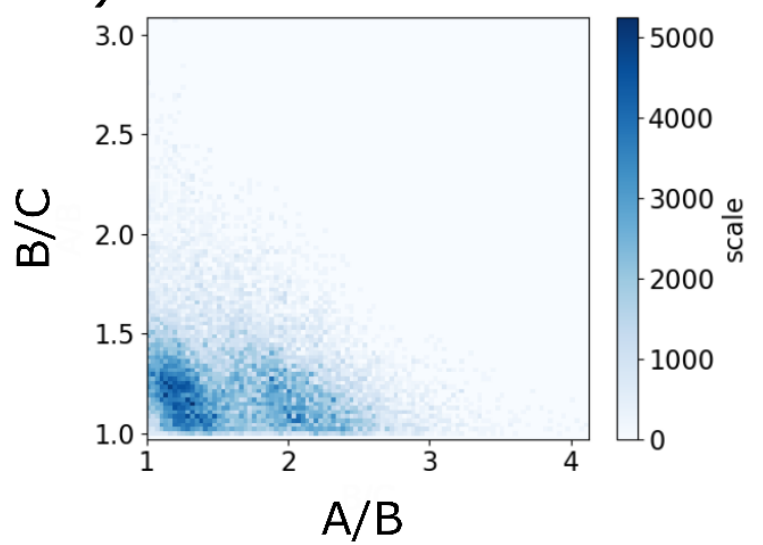

b)

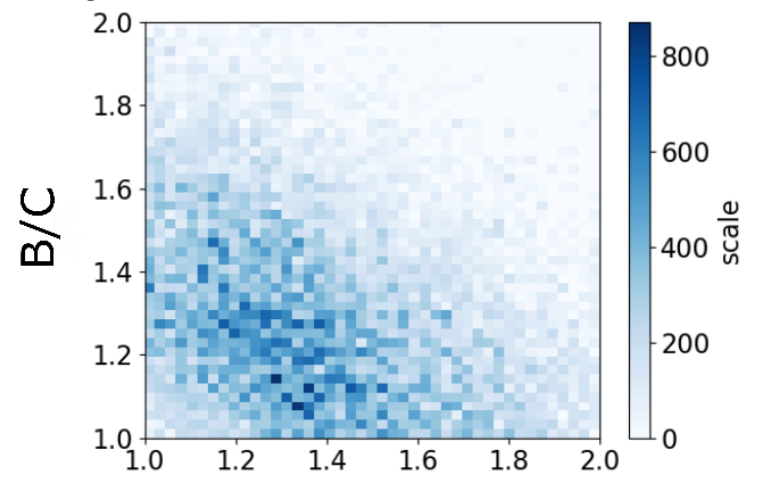

d)

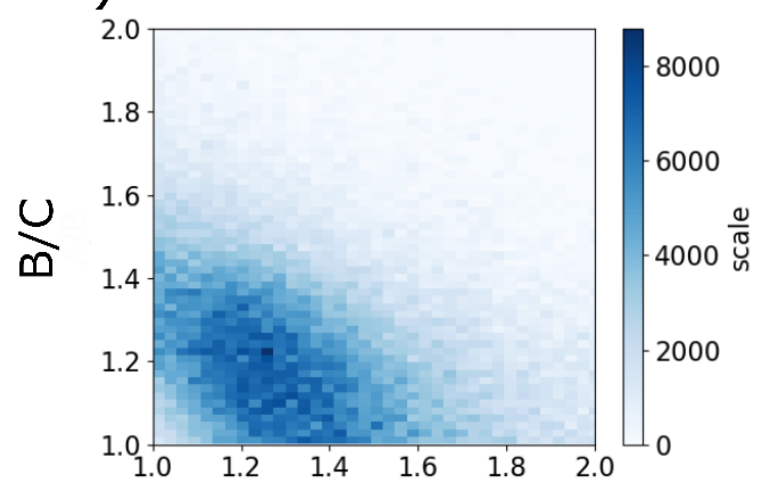

f)

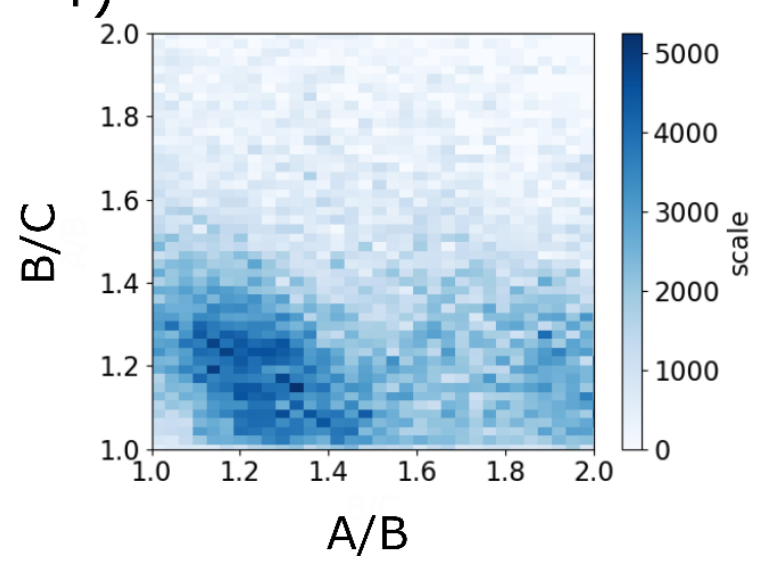

Figure S5: Histograms for DEA systems. a-b) DEA8 histograms. c-d) DEA10 histograms. e-f) DEA12 histograms. 


\section{MEGA histograms}

a)

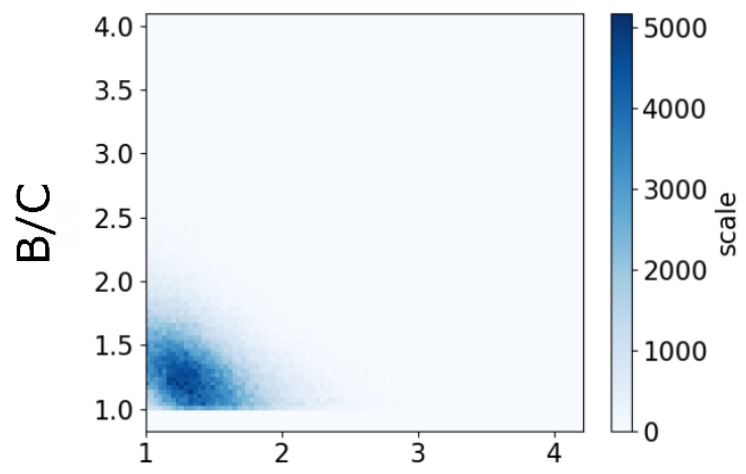

c)

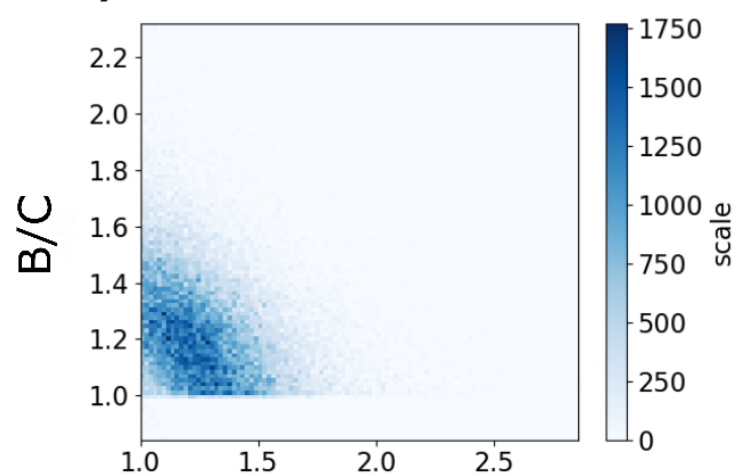

e)

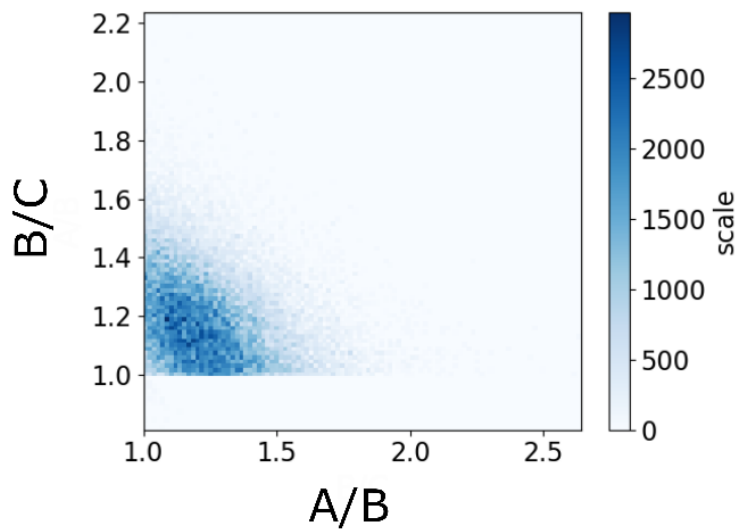

b)

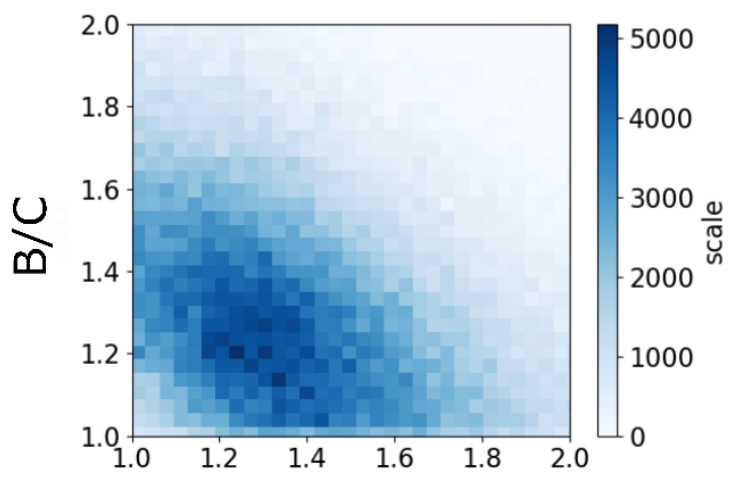

d)

A/B
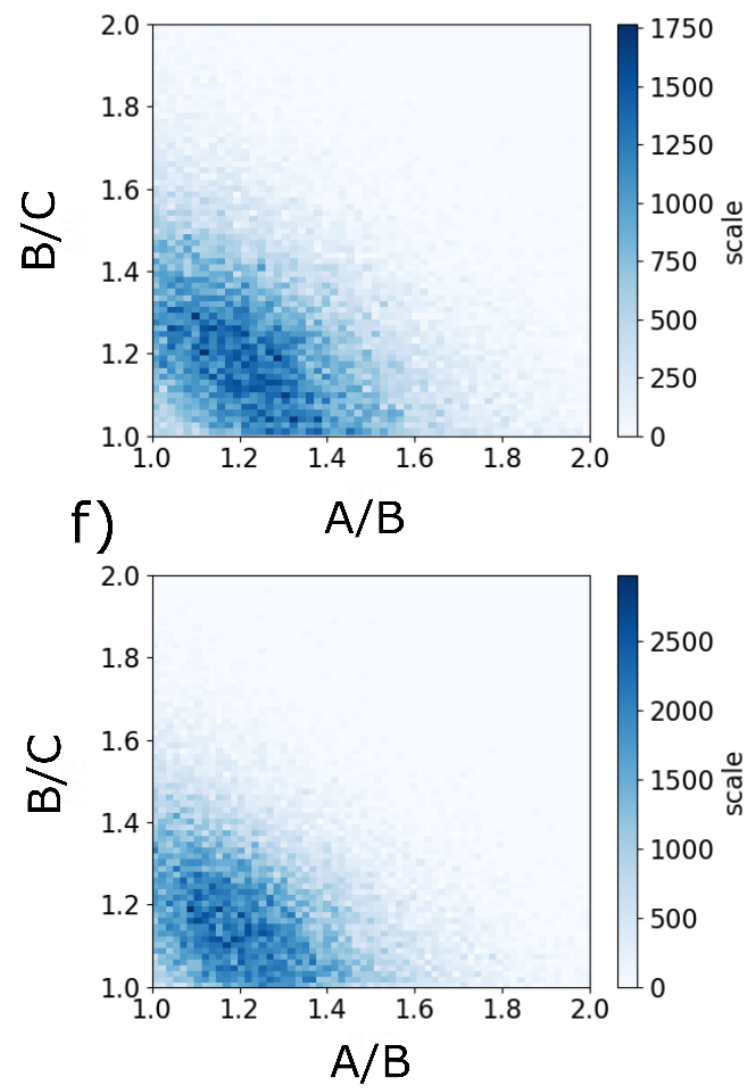

Figure S6: Histograms for MEGA systems. a-b) MEGA8 histograms. c-d) MEGA10 histograms. e-f) MEGA12 histograms. 


\section{HEGA histograms}

a)

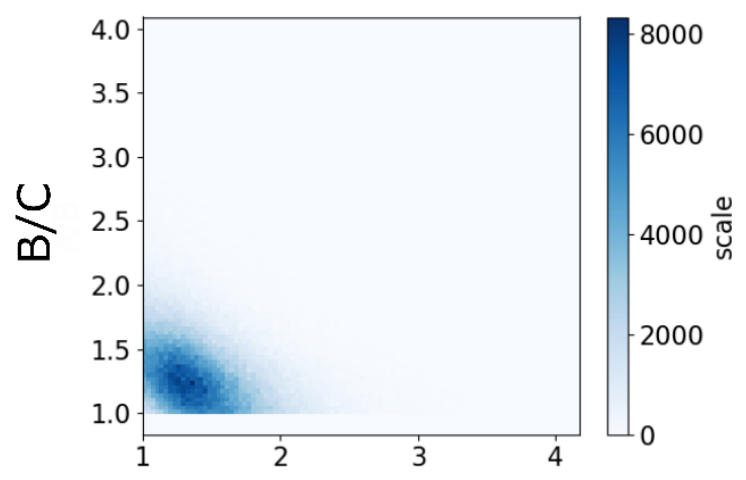

c) $\quad A / B$

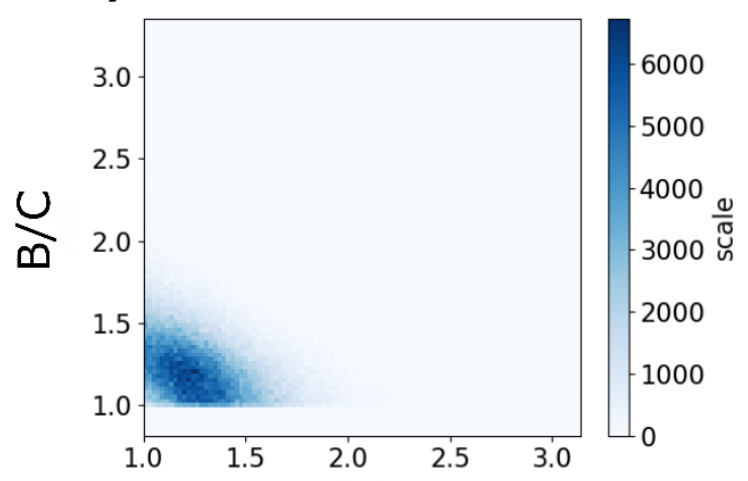

e)

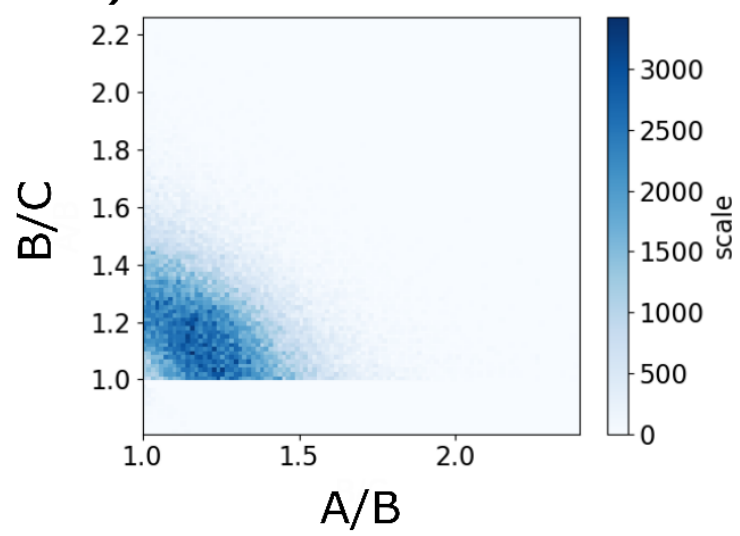

b)

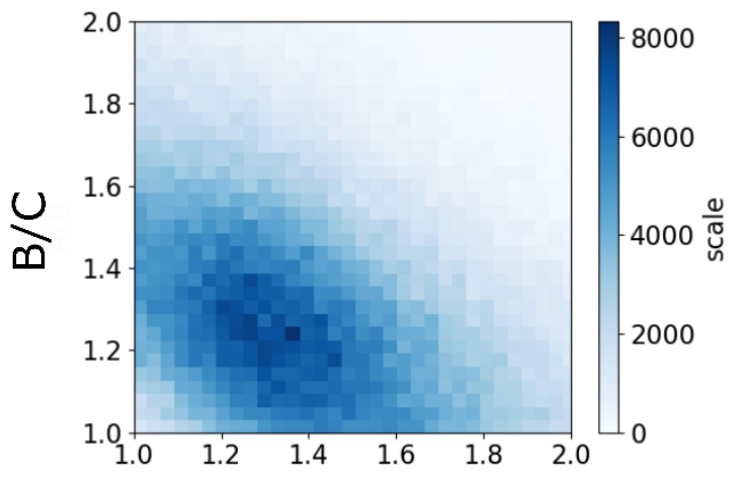

d)

A/B
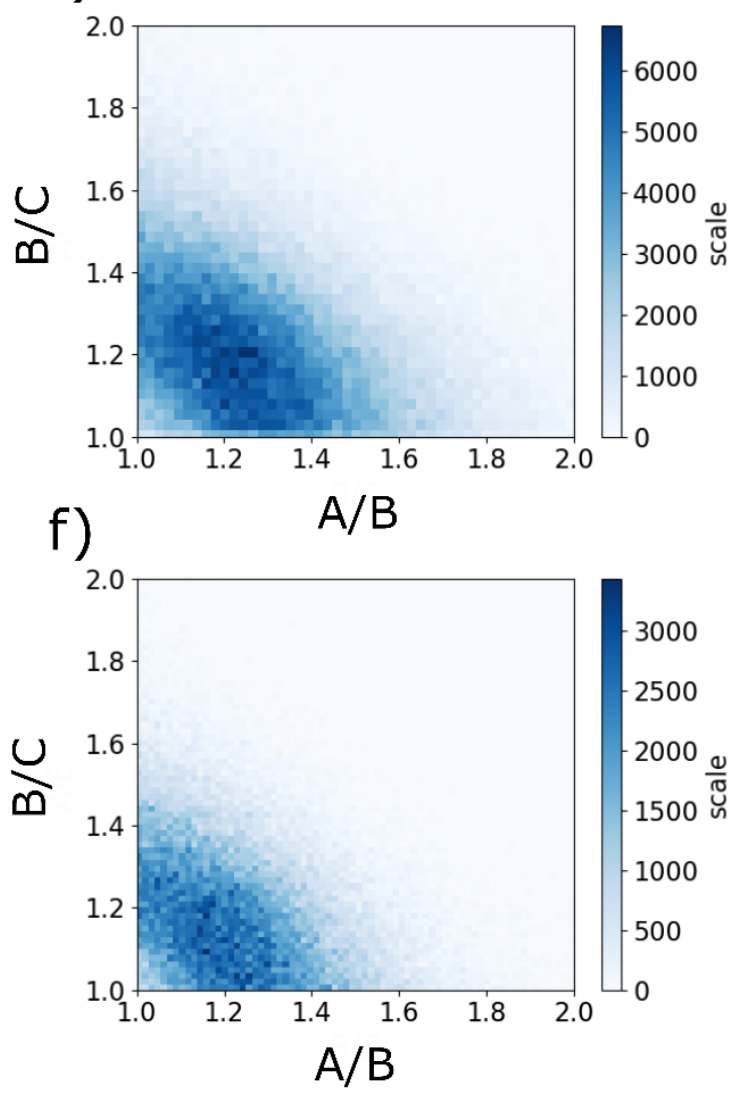

Figure S7: Histograms for HEGA systems. a-b) HEGA8 histograms. c-d) HEGA10 histograms. e-f) HEGA12 histograms. 
a)

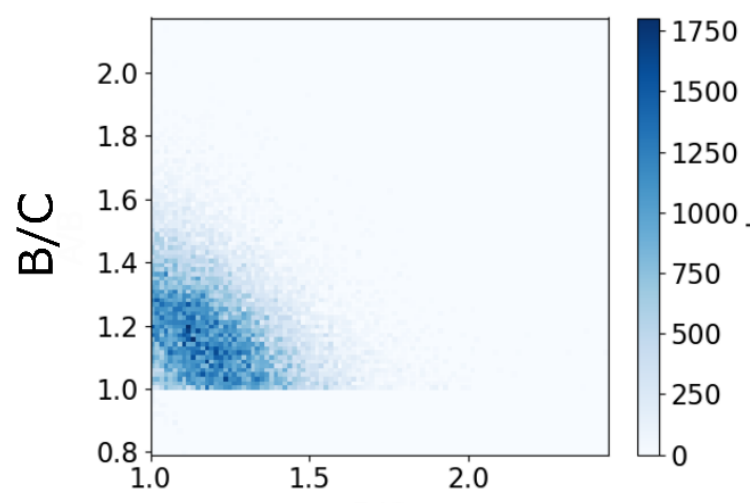

c)

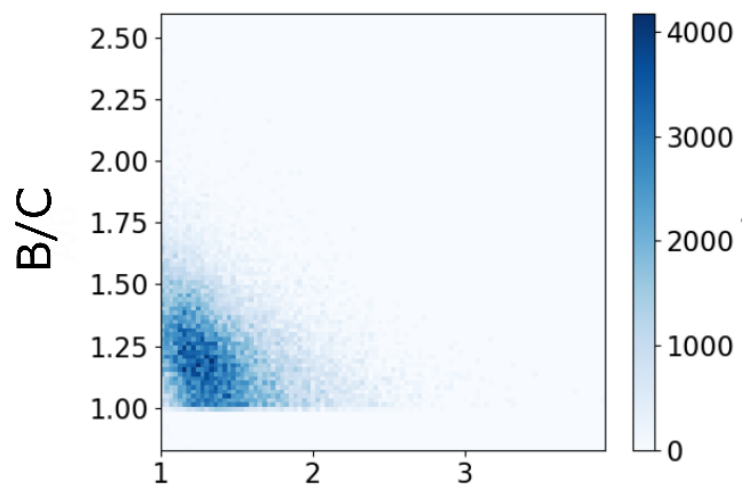

e)

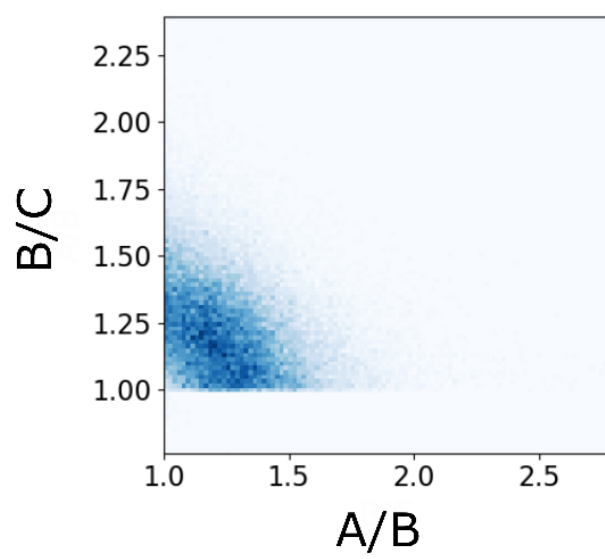

b)

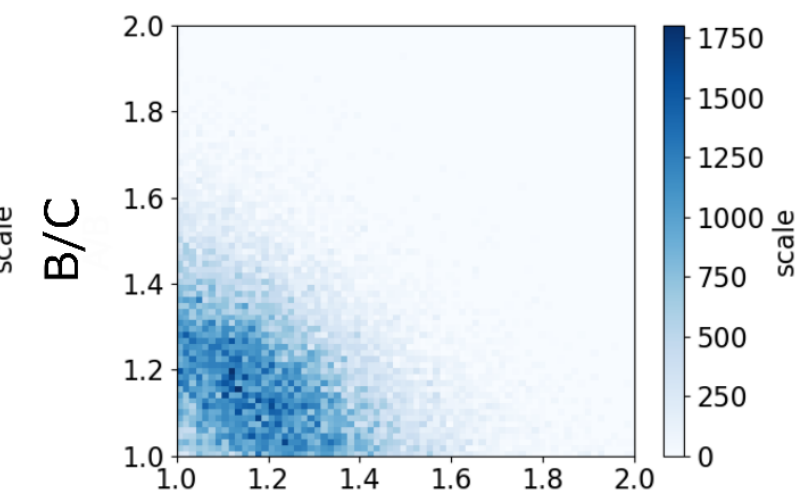

d)

$\mathrm{A} / \mathrm{B}$

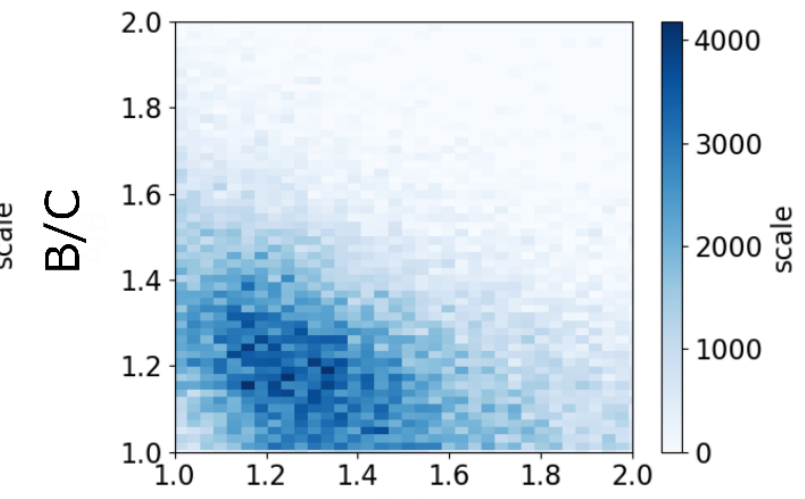

f)

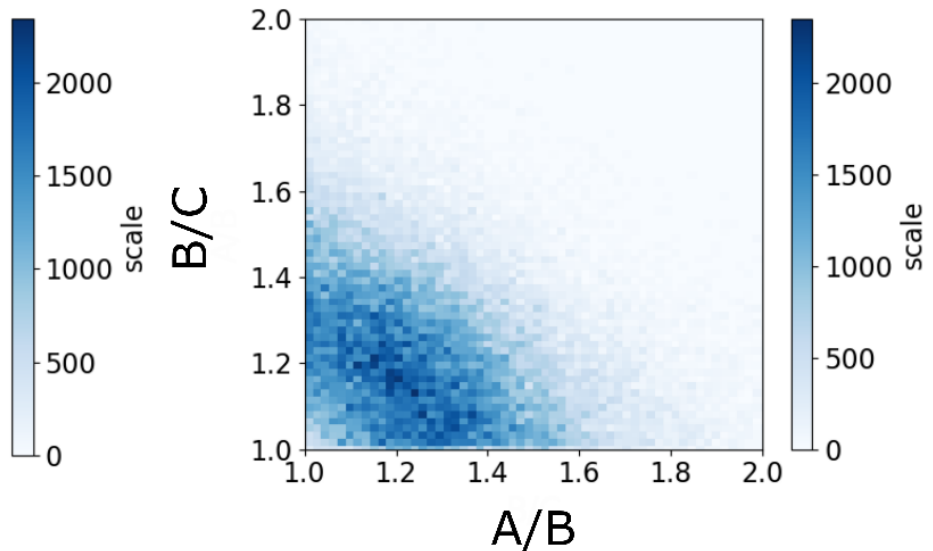

Figure S8: Histograms for TEDA12, CYGLU and CYMAL systems. a-b) TEDA12 histograms. c-d) CYGLU4 histograms. e-f) CYMAL4 histograms. 


\section{GLUCO histograms}

a)

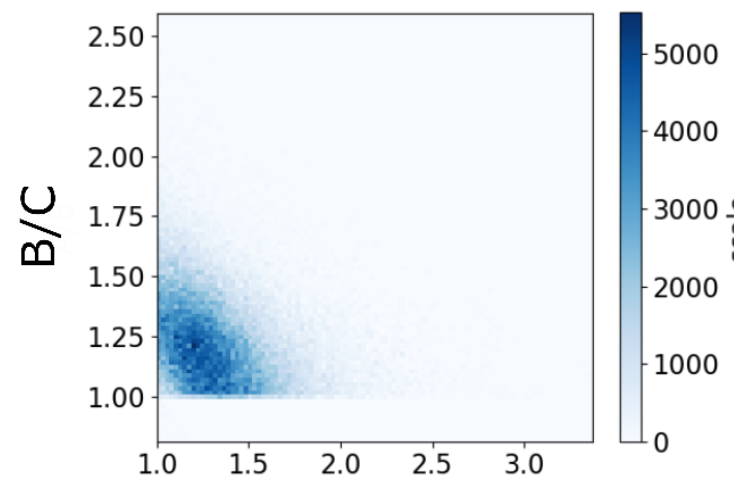

c)

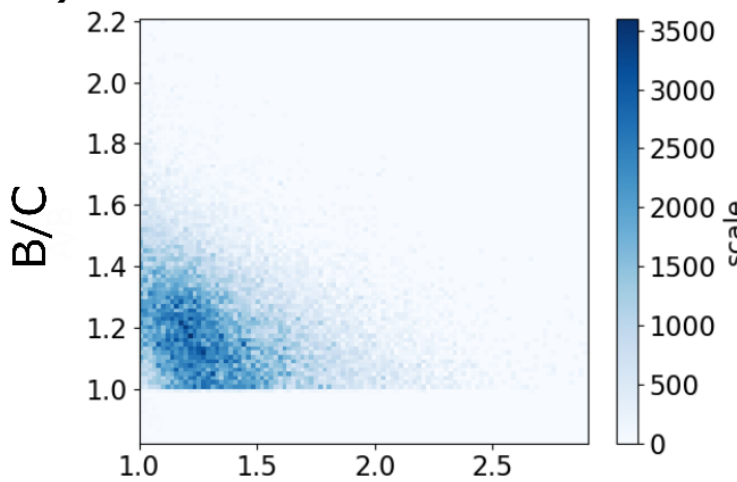

e)

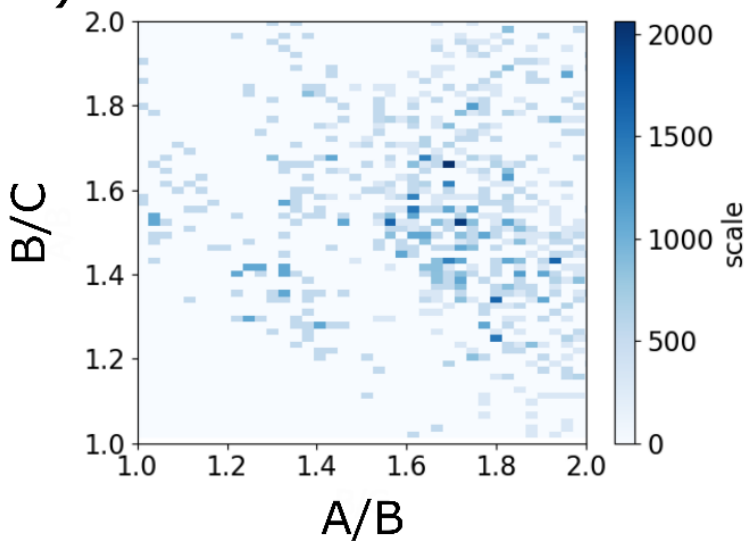

b)

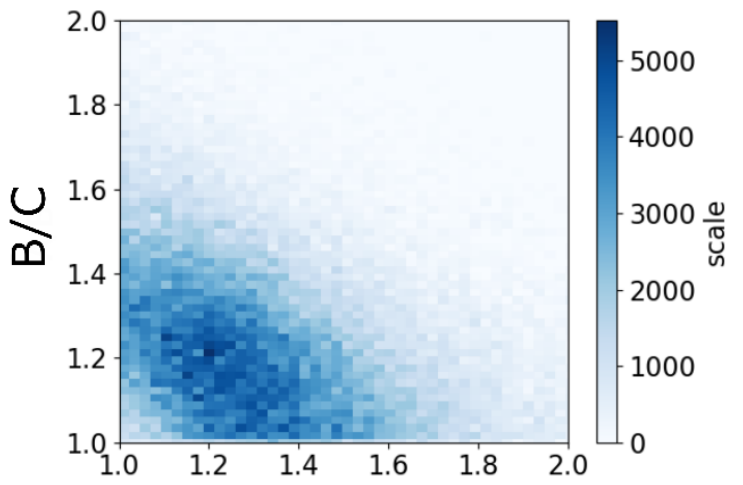

d)

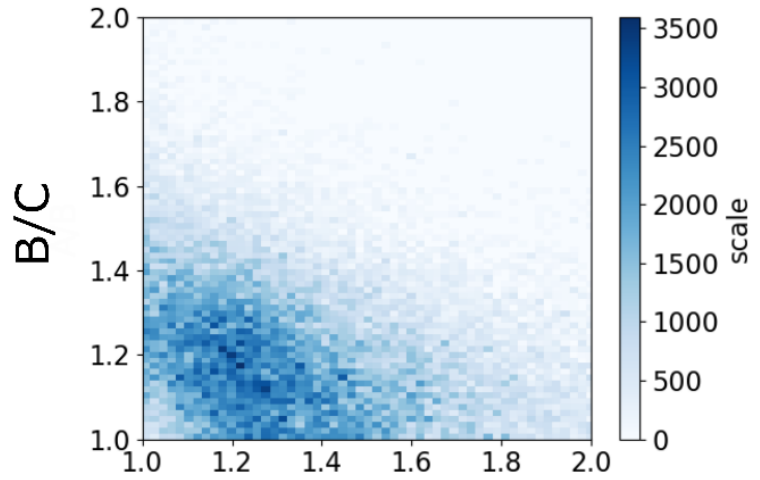

f)

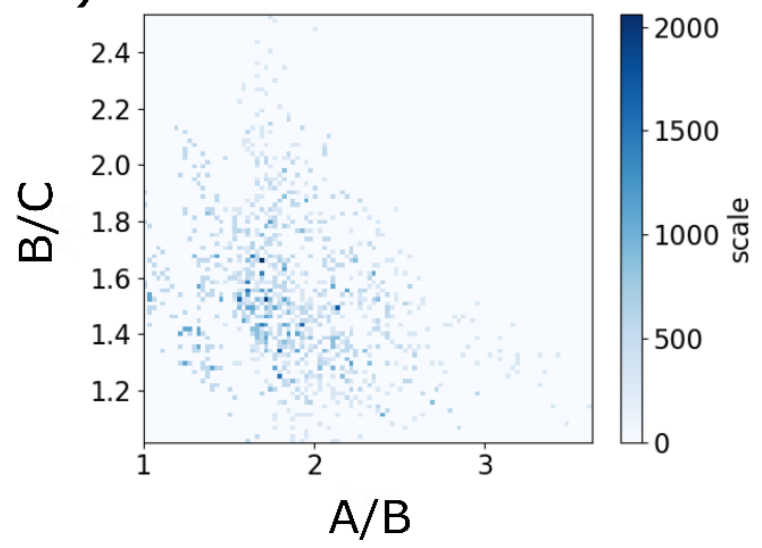

Figure S9: Histograms for GLUCO systems. a-b) GLUCO8 histograms. c-d) GLUCO10 histograms. e-f) GLUCO12 histograms. 
a)

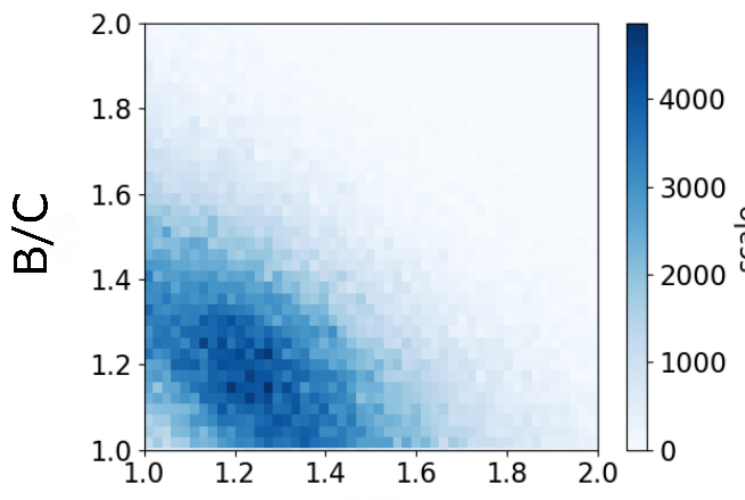

c)

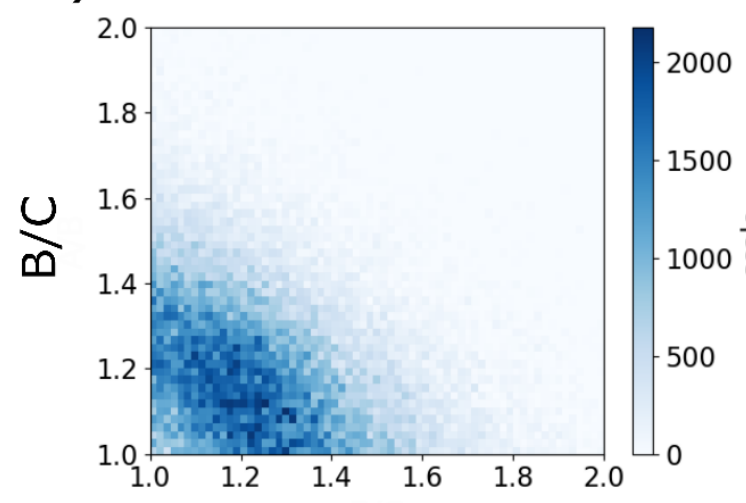

e)

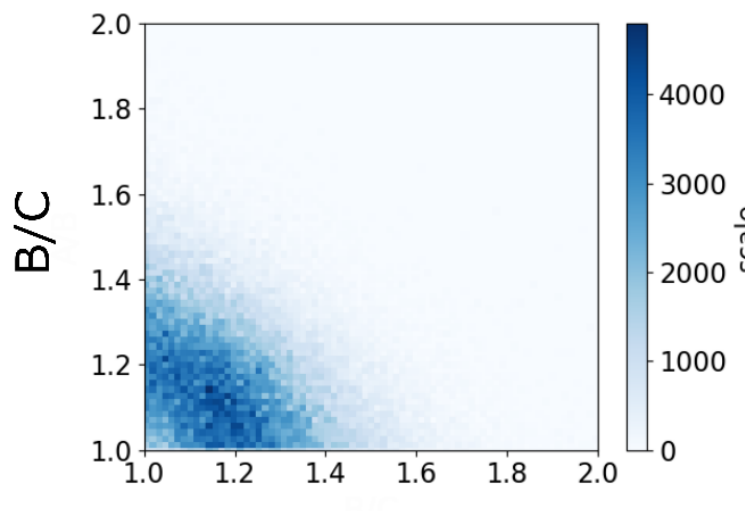

A/B b)

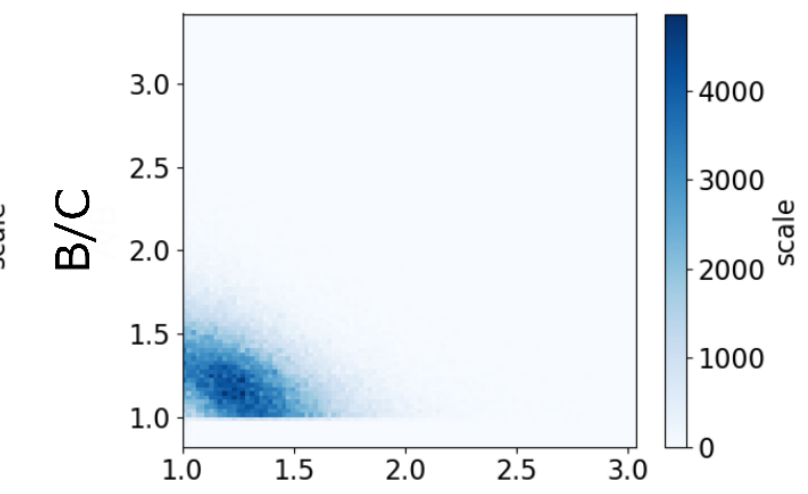

d)

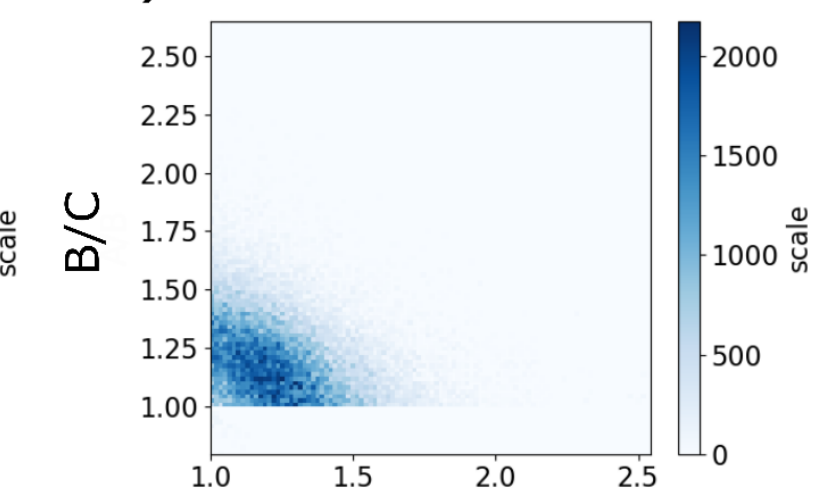

f)

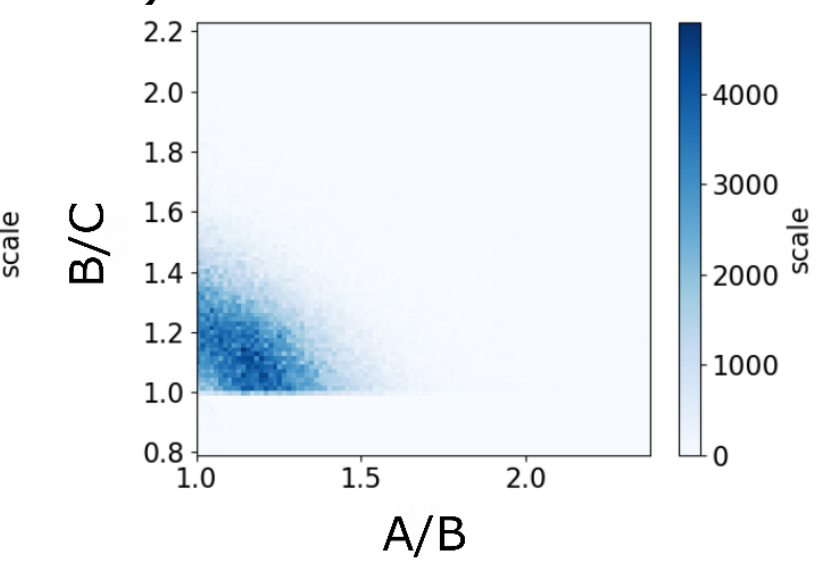

Figure S10: Histograms for MALTO systems. a-b) MALTO8 histograms. c-d) MALTO10 histograms. e-f) MALTO12 histograms. 


\section{Parameters for Bonded Interactions}

\section{Bead distance calculator script}

An open access script was developed to automatise the bead-bead distance calculation. The script can accept either the molecule SMILES, then it performs and show the optimisation or it can use directly an optimised structure in mol 2 format. After selecting the molecule fragment used in the bead description and providing the necessary parameters $\left(a_{i j}, R_{i j}, r_{i j}\right.$ and $\left.k_{s}\right)$ the script returns the $r_{0}$. The script is freely available and it can be found at:

https://gitlab.developers.cam.ac.uk/el446/bead distance calculator.

\section{Bead-bead distance comparison between methods}

The reference method consist in the following steps:

- The distances between different fragments were obtained using Molecular Mechanics and translated into DPD units.

- DPD simulations were run with different equilibrium distances and the radial distributions of the two beads were collected.

- The average distance value of the two beads during the DPD simulation was obtained from the weighted average of the radial distribution.

- The values were used as benchmarks for the previous parameterisation method.

A comparison between the two methods is reported in table S1. 
Table S1: Fragment distances obtained from MM optimisation, input distance obtained from the recursive (Method 1) and the new method (Method 2) for a selected number of pairwise bead interactions.

\begin{tabular}{|c|c|c|c|c|c|}
\hline Surfactant & $\begin{array}{l}\text { Bear } \\
\text { pair }\end{array}$ & $\begin{array}{l}\mathrm{MM} \\
\text { distance }\end{array}$ & $\begin{array}{l}\text { Method } \\
\text { 1: Input } \\
\text { distance }\end{array}$ & $\begin{array}{l}\text { Method } \\
\text { 2: Input } \\
\text { distance }\end{array}$ & Difference \\
\hline MEA & $\begin{array}{l}\text { AM2 } \\
-\mathrm{C} 2\end{array}$ & 0.65 & 0.60 & 0.58 & 0.02 \\
\hline MEA & $\begin{array}{l}\text { AM2 } \\
-\mathrm{OH}\end{array}$ & $\begin{array}{l}0.67 / 0 . \\
68\end{array}$ & 0.65 & $\begin{array}{l}0.64 / 0.6 \\
5\end{array}$ & $0.00 / 0.01$ \\
\hline DEA & $\begin{array}{l}\text { AM3' } \\
-\mathrm{C} 2\end{array}$ & 0.67 & 0.64 & 0.62 & 0.02 \\
\hline DEA & $\begin{array}{l}\text { AM3' } \\
-\mathrm{OH} 1\end{array}$ & 0.67 & 0.66 & 0.65 & 0.01 \\
\hline HEGA & $\begin{array}{l}\text { AM3' } \\
-\mathrm{C} 2\end{array}$ & 0.67 & 0.64 & 0.62 & 0.02 \\
\hline HEGA & $\begin{array}{l}\text { AM3' } \\
-\mathrm{OH} 1\end{array}$ & 0.67 & 0.66 & 0.65 & 0.01 \\
\hline HEGA & $\begin{array}{l}\text { AM3' } \\
-\mathrm{OH}^{\prime}\end{array}$ & 0.67 & 0.66 & 0.65 & 0.01 \\
\hline MEGA & $\begin{array}{l}\text { AM3 } \\
-\mathrm{OH}^{\prime}\end{array}$ & 0.67 & 0.65 & 0.65 & 0.00 \\
\hline MEGA & $\begin{array}{l}\text { AM3 } \\
-\mathrm{C} 2\end{array}$ & 0.68 & 0.64 & 0.63 & 0.01 \\
\hline TEDA & $\begin{array}{l}\text { AM2 } \\
\text {-EO }\end{array}$ & 0.78 & 0.75 & 0.74 & 0.01 \\
\hline XYL & $\begin{array}{l}\text { ES- } \\
\mathrm{OH}^{\prime}\end{array}$ & 0.65 & 0.63 & 0.61 & 0.02 \\
\hline XYL & $\begin{array}{l}\mathrm{OH}^{\prime}- \\
\mathrm{OH}^{\prime}\end{array}$ & 0.44 & 0.42 & 0.40 & 0.02 \\
\hline Tail & $\begin{array}{l}\mathrm{C} 2- \\
\mathrm{C} 2\end{array}$ & 0.455 & 0.39 & 0.38 & 0.01 \\
\hline Tail & $\mathrm{C} 2-\mathrm{T}$ & 0.35 & 0.29 & 0.26 & 0.03 \\
\hline
\end{tabular}




\section{GLY8 Parameterisation}
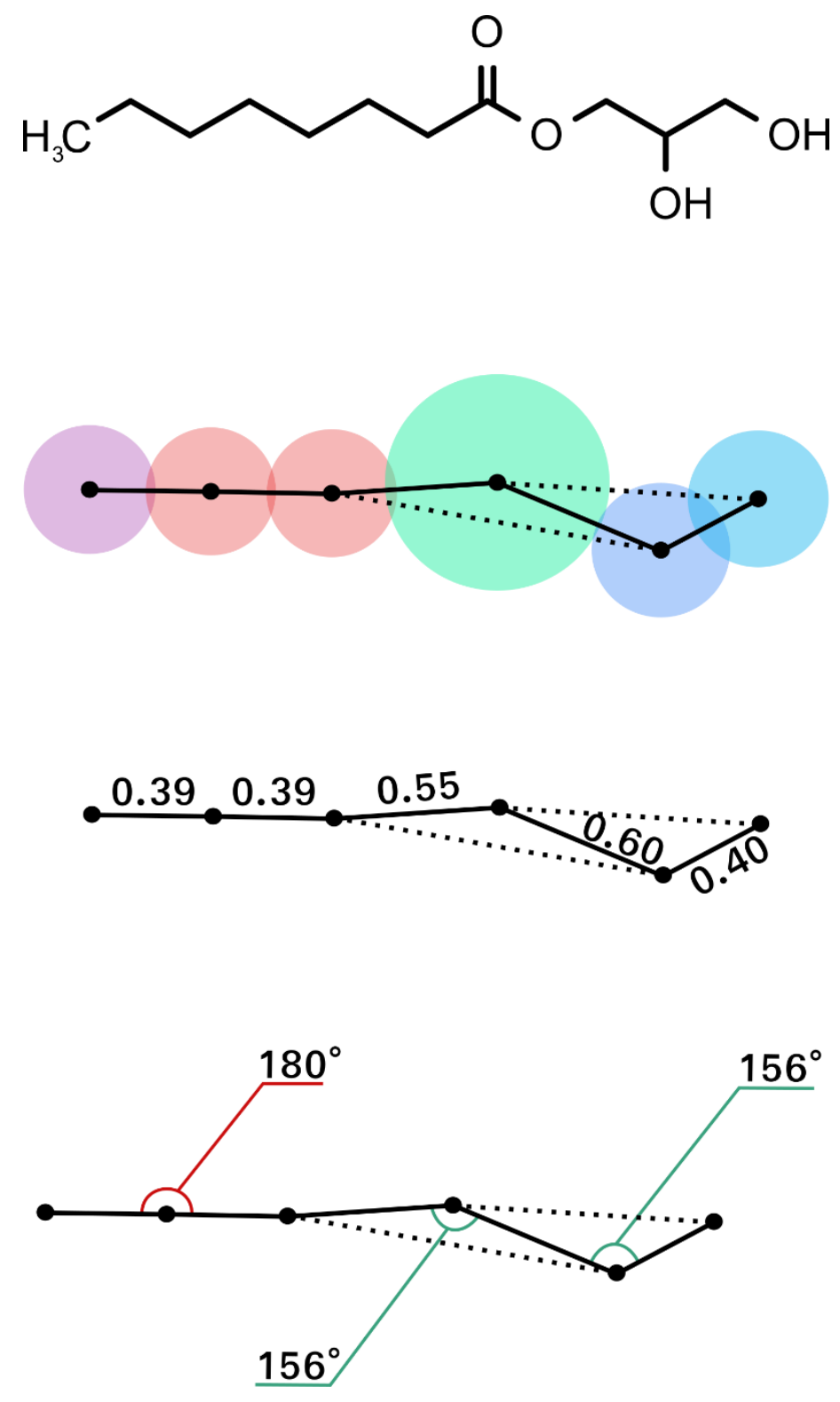

Figure S11: Parameterisation of GLY8: a) shows the 2D surfactant structure, b) shows the GC description with the full lines representing the 1-2 bonds and the dashed lines the 1-3 angles. c) Shows the values for the 1-2 bead distances obtained using equation 11 and and d) shows the 1-3 angles obtained from the MM optimised structure. 


\section{XYL8 Parameterisation}<smiles>CCCCCCCC(=O)OCC(O)C(O)C(O)CO</smiles>
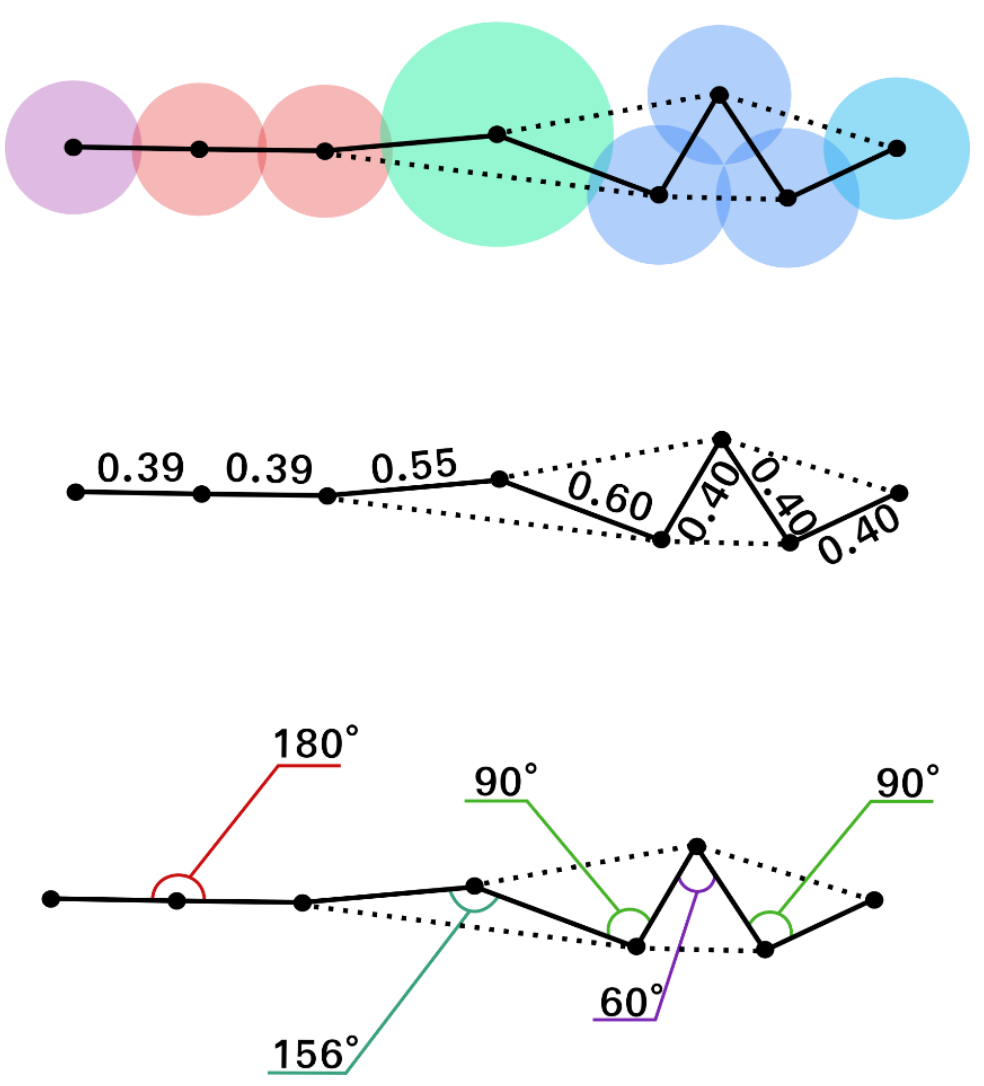

Figure S12: Parameterisation of XYL8: a) shows the 2D surfactant structure, b) shows the GC description with the full lines representing the 1-2 bonds and the dashed lines the 1-3 angles. c) Shows the values for the 1-2 bead distances obtained using equation 11 and d) shows the 1-3 angles obtained from the MM optimised structure. 

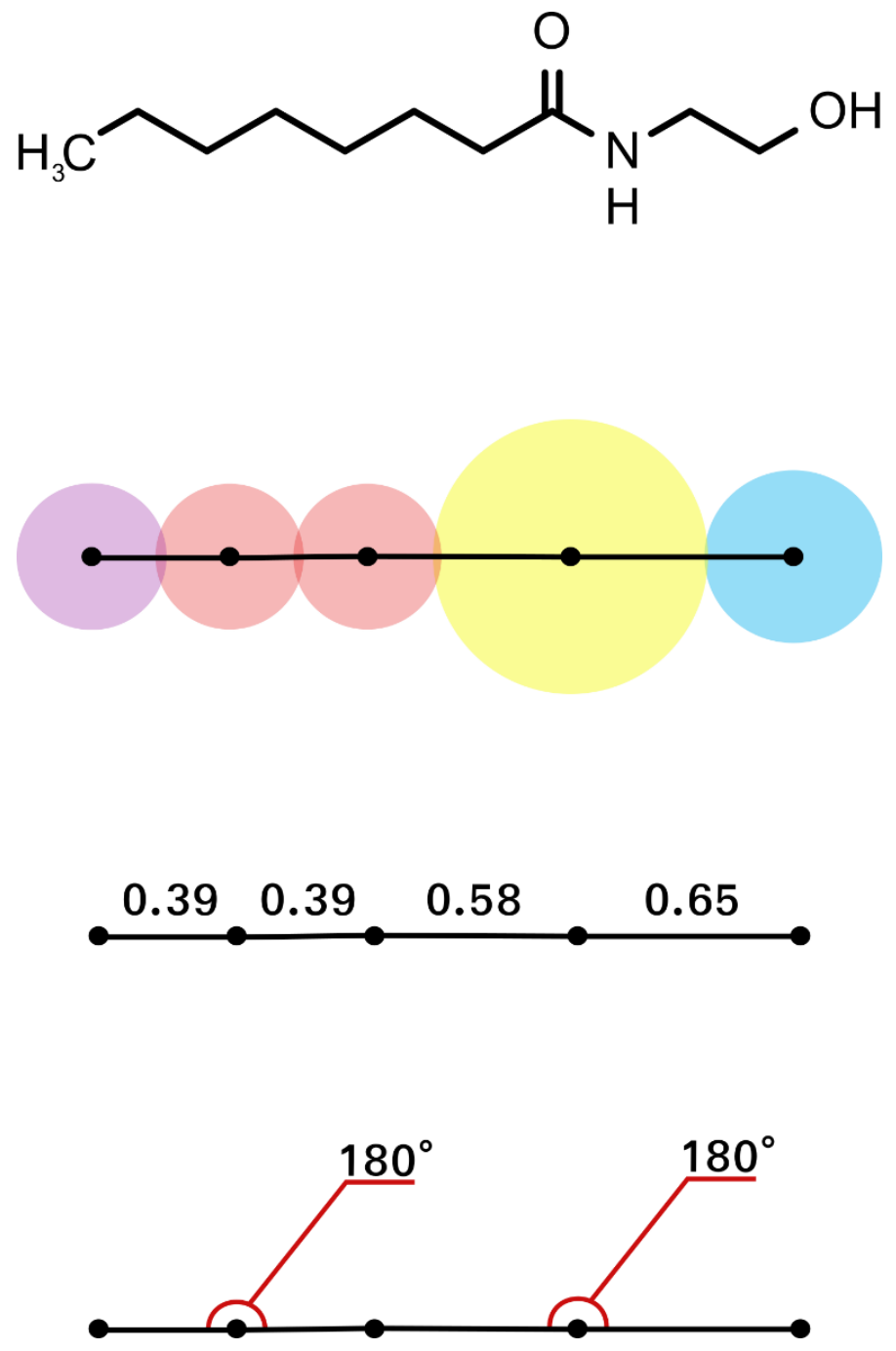

Figure S13: Parameterisation of MEA8: a) shows the 2D surfactant structure, b) shows the GC description with the full lines representing the 1-2 bonds and the dashed lines the 1-3 angles. c) Shows the values for the 1-2 bead distances obtained using equation 11 and d) shows the 1-3 angles obtained from the MM optimised structure. 

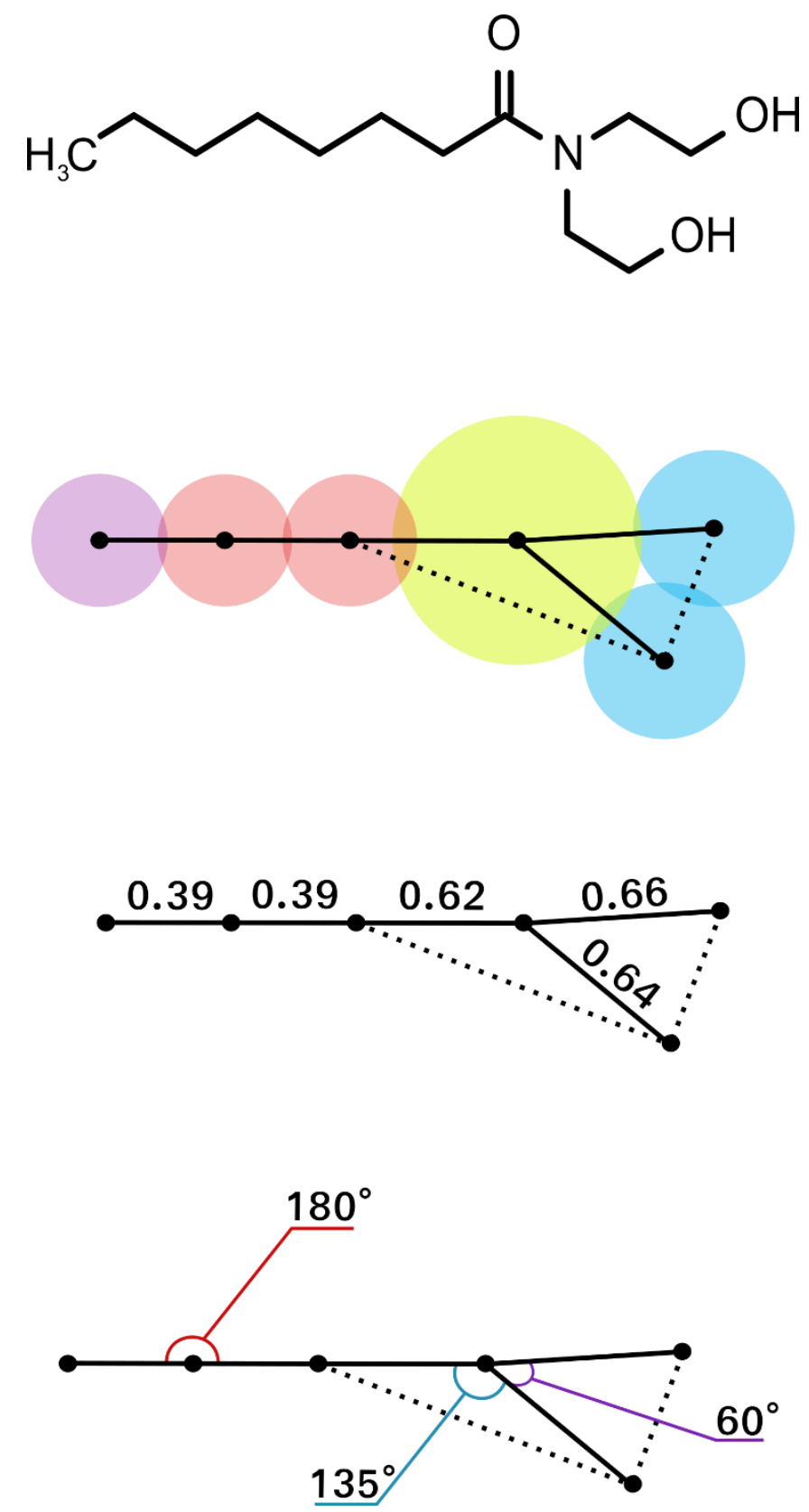

Figure S14: Parameterisation of DEA8: a) shows the 2D surfactant structure, b) shows the GC description with the full lines representing the 1-2 bonds and the dashed lines the 1-3 angles. c) Shows the values for the 1-2 bead distances obtained using equation 11 and d) shows the 1-3 angles obtained from the MM optimised structure. 

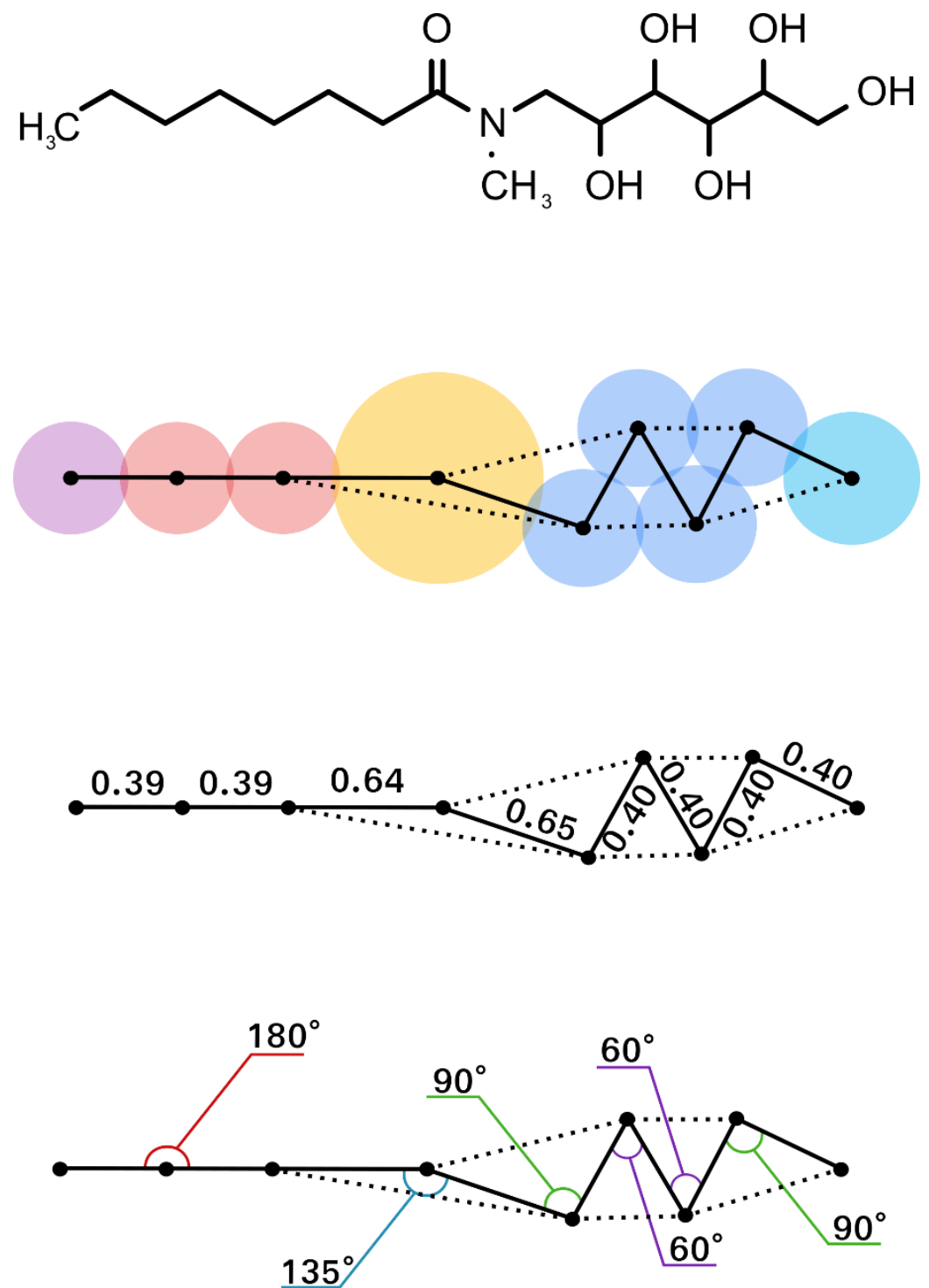

Figure S15: Parameterisation of MEGA8: a) shows the 2D surfactant structure, b) shows the GC description with the full lines representing the 1-2 bonds and the dashed lines the 1-3 angles. c) Shows the values for the 1-2 bead distances obtained using equation 11 and d) shows the 1-3 angles obtained from the MM optimised structure. 

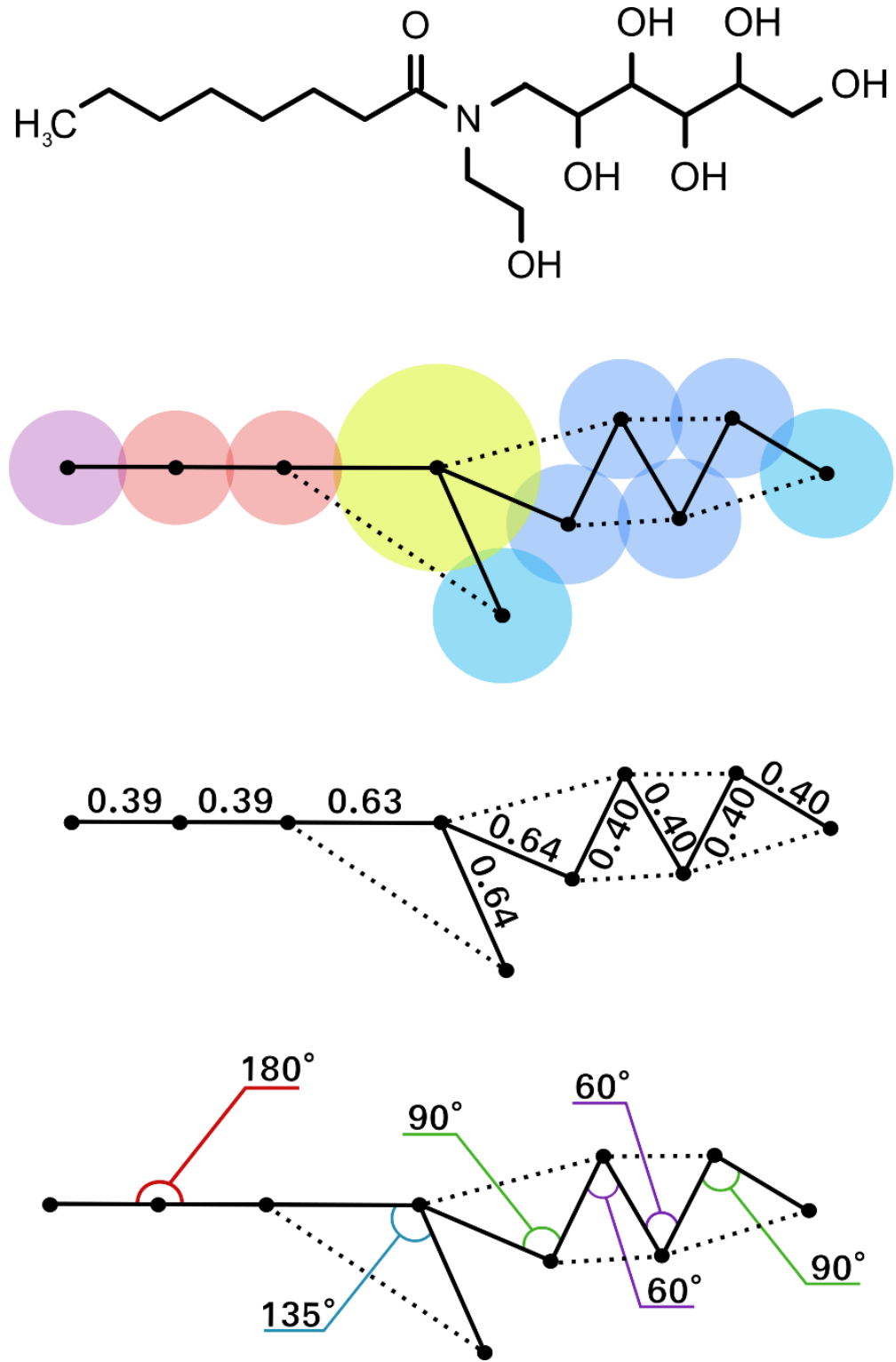

Figure S16: Parameterisation of HEGA8: a) shows the 2D surfactant structure, b) shows the GC description with the full lines representing the 1-2 bonds and the dashed lines the 1-3 angles. c) Shows the values for the 1-2 bead distances obtained using equation 11 and d) shows the 1-3 angles obtained from the MM optimised structure. 

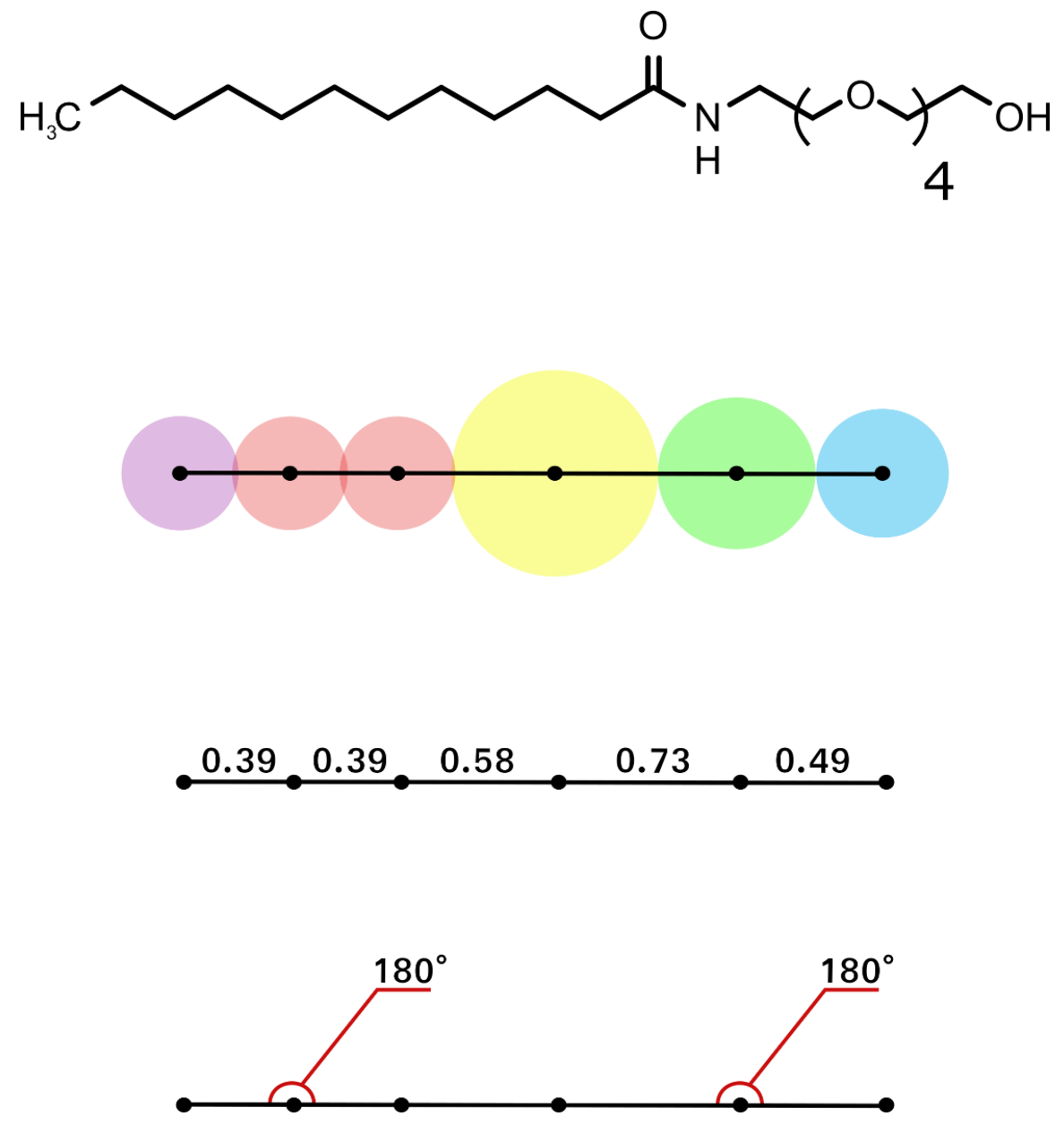

Figure S17: Parameterisation of TEDA12: a) shows the 2D surfactant structure, b) shows the GC description with the full lines representing the 1-2 bonds and the dashed lines the 1-3 angles. c) Shows the values for the 1-2 bead distances obtained using equation 11 and d) shows the 1-3 angles obtained from the MM optimised structure. 

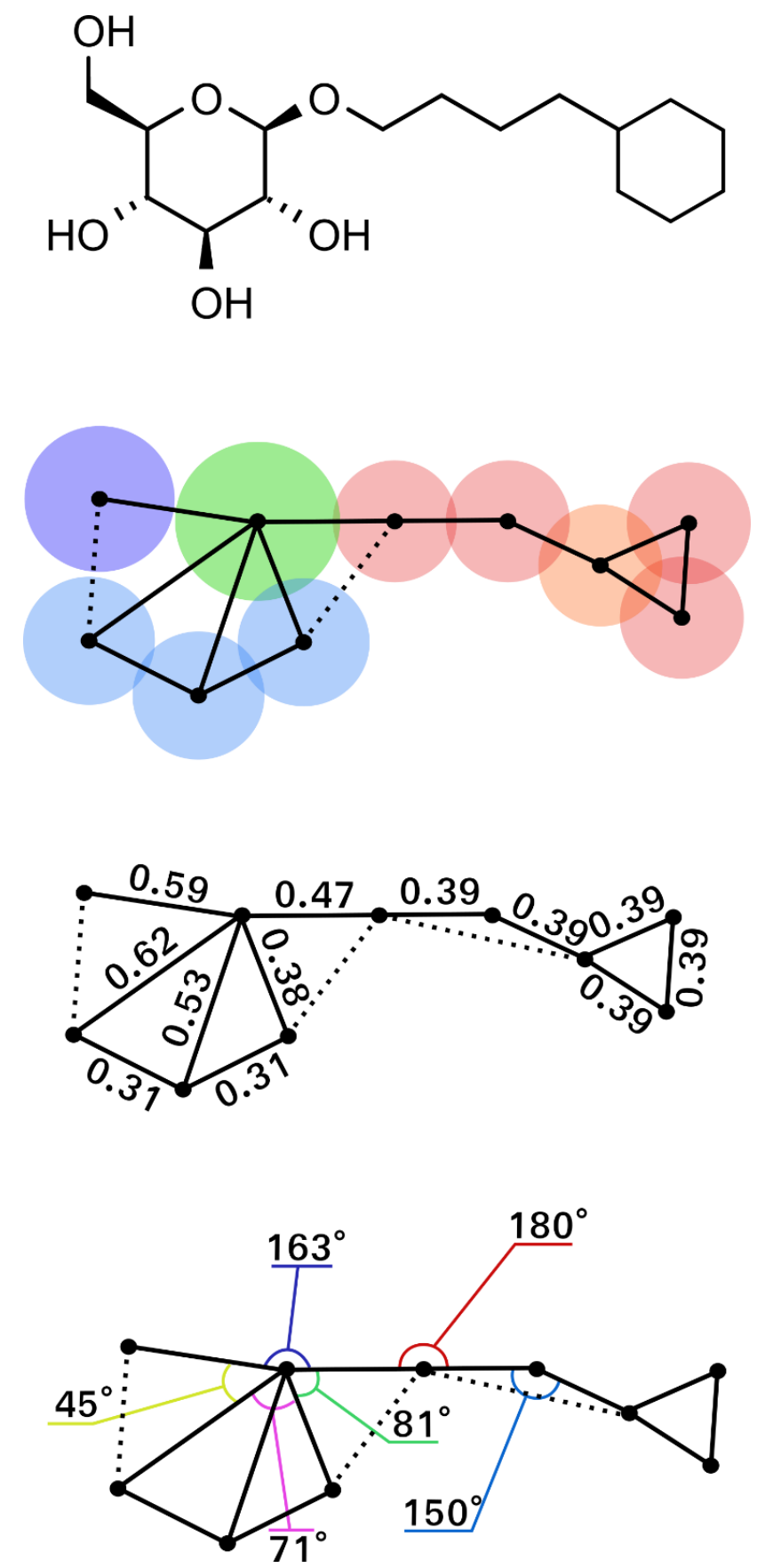

Figure S18: Parameterisation of CYGLU4: a) shows the 2D surfactant structure, b) shows the GC description with the full lines representing the 1-2 bonds and the dashed lines the 1-3 angles. c) Shows the values for the 1-2 bead distances obtained using equation 11 and d) shows the 1-3 angles obtained from the MM optimised structure. 


\section{CYMAL4 Parameterisation}<smiles>OC[C@H]1OC(O[C@@H]2[C@@H](CO)O[C@@H](OCCCCC3CCCCC3)[C@@H](O)[C@H]2O)[C@H](O)[C@@H](O)[C@@H]1O</smiles>
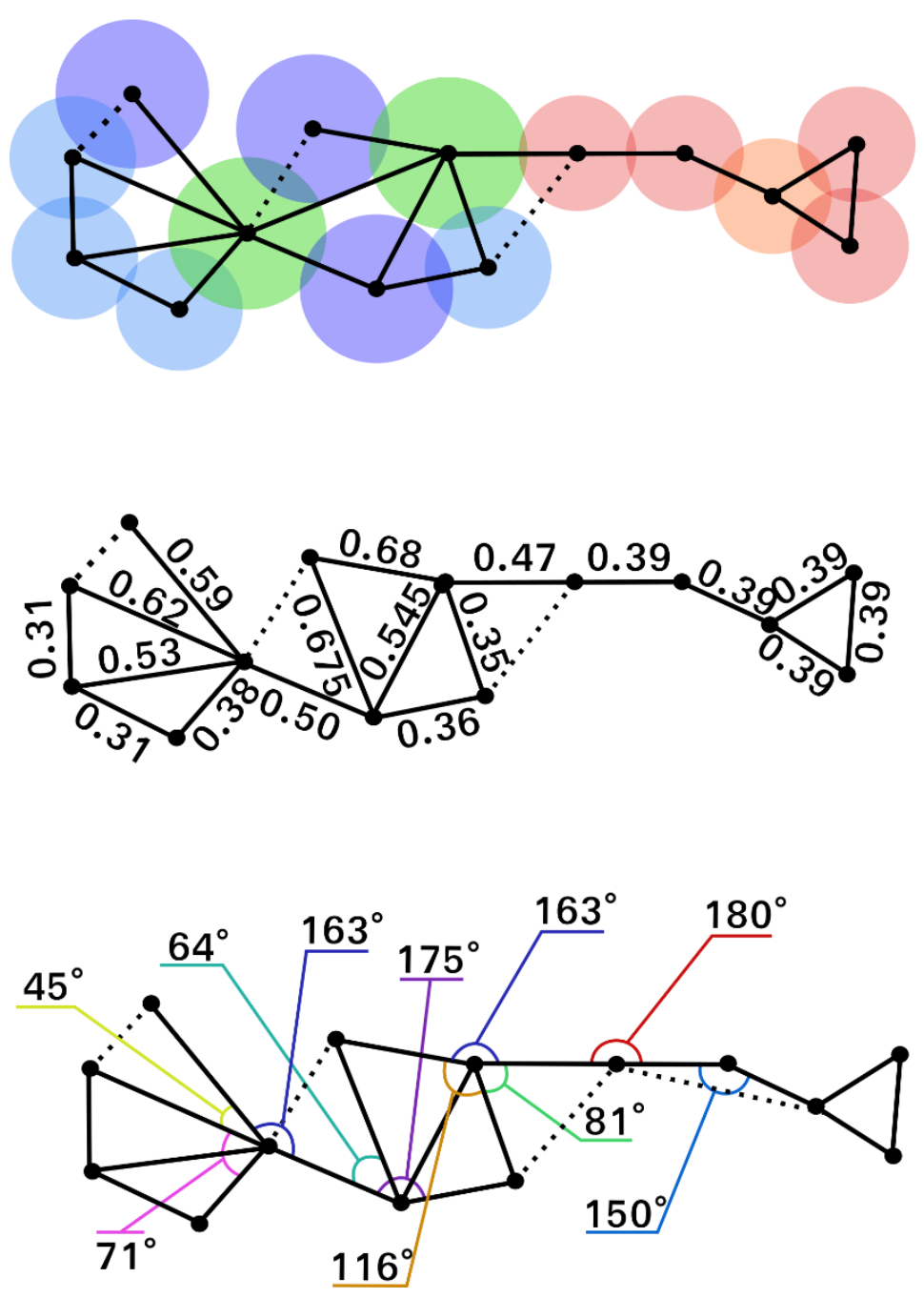

Figure S19: Parameterisation of CYMAL4: a) shows the 2D surfactant structure, b) shows the GC description with the full lines representing the 1-2 bonds and the dashed lines the 1-3 angles. c) Shows the values for the 1-2 bead distances obtained using equation 11 and d) shows the 1-3 angles obtained from the MM optimised structure. 


\section{GLUC08 Parameterisation}
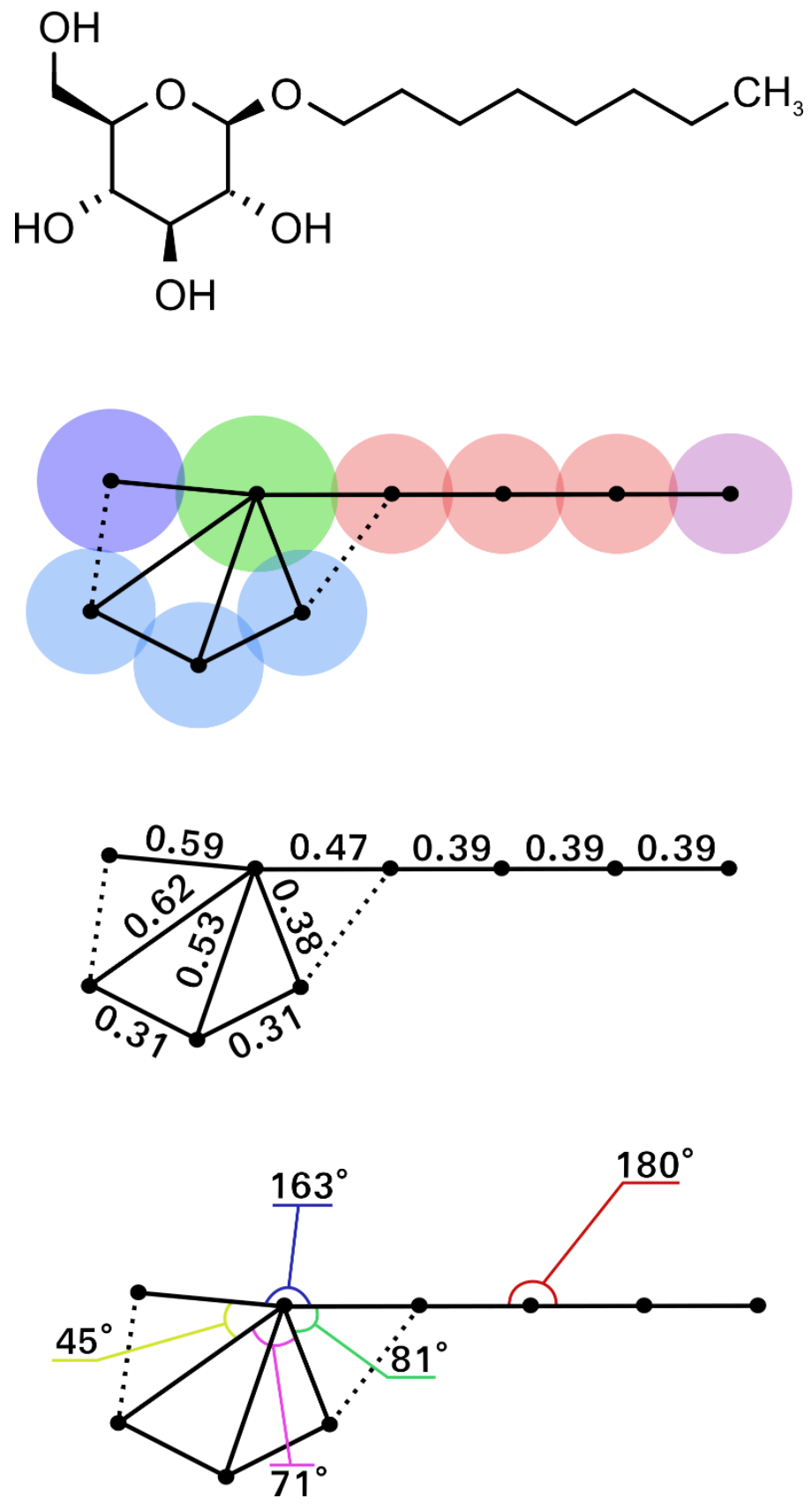

Figure S20: Parameterisation of GLU8: a) shows the 2D surfactant structure, b) shows the GC description with the full lines representing the 1-2 bonds and the dashed lines the 1-3 angles. c) Shows the values for the 1-2 bead distances obtained using equation 11 and d) shows the 1-3 angles obtained from the MM optimised structure. 


\section{MALT08 Parameterisation}<smiles>CCCCCCCCO[C@H]1O[C@H](CO)[C@@H](OC2O[C@H](CO)[C@@H](O)[C@H](O)[C@H]2O)[C@H](O)[C@H]1O</smiles>
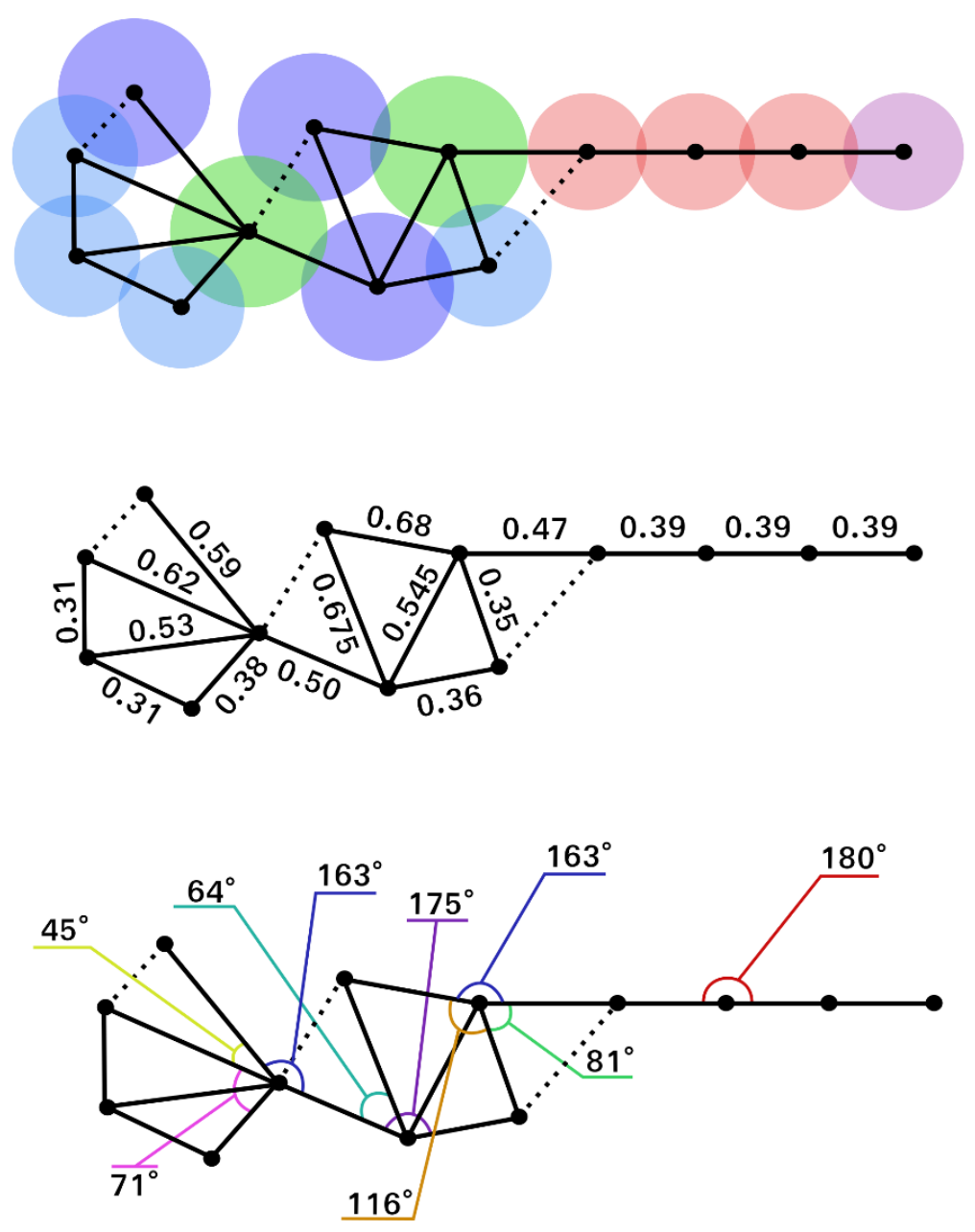

Figure S21: Parameterisation of MAL8: a) shows the 2D surfactant structure, b) shows the GC description with the full lines representing the 1-2 bonds and the dashed lines the 1-3 angles. c) Shows the values for the 1-2 bead distances obtained using equation 11 and d) shows the 1-3 angles obtained from the MM optimised structure. 


\section{Amide Conformers}

Table S2: Calculated and experimental values of CMC and Nagg.

\begin{tabular}{l|l|l|l|l|l}
\hline Surfactant & Amide & $\begin{array}{l}\text { CMC/mM } \\
\text { calc. }\end{array}$ & $\begin{array}{l}N_{\text {agg }} \\
\text { calc. }\end{array}$ & $\begin{array}{l}\text { CMC/mM } \\
\text { exp. }\end{array}$ & $\begin{array}{l}N_{\text {agg }} \\
\text { exp. }\end{array}$ \\
\hline MEGA8 & trans & $101.03 \pm 3.56$ & $9-18$ & $51-79$ & $24-85$ \\
MEGA10 & cis & $112.32 \pm 4.26$ & $9-12$ & & \\
& trans & $9.44 \pm 0.45$ & $26-28$ & $4-7$ & $28-75$ \\
MEGA12 & cis & $11.03 \pm 0.52$ & $24-27$ & & \\
& trans & $1.02 \pm 0.11$ & $26-36$ & 0.35 & - \\
\hline
\end{tabular}

